\title{
Constraining the mass transfer in massive binaries through progenitor evolution models of Wolf-Rayet+O binaries
}

\author{
J. Petrovic ${ }^{1,2}$, N. Langer ${ }^{1}$, and K. A. van der Hucht ${ }^{3,4}$ \\ 1 Sterrenkundig Instituut, Universiteit Utrecht, Princetonplein 5, 3584 CC Utrecht, The Netherlands \\ e-mail: petrovic@astro.uu.nl \\ 2 Astronomical Institute, Radboud Universiteit Nijmegen, Toernooiveld 1, 6525 ED, Nijmegen, The Netherlands \\ ${ }^{3}$ SRON, Nationaal Instituut voor Ruimte Onderzoek, Sorbonnelaan 2, 3584 CA Utrecht, The Netherlands \\ 4 Sterrenkundig Instituut Anton Pannekoek, Universiteit van Amsterdam, Kruislaan 403, 1098 SJ Amsterdam, The Netherlands
}

Received 15 November 2004 / Accepted 18 February 2005

\begin{abstract}
Since close WR+O binaries are the result of a strong interaction of both stars in massive close binary systems, they can be used to constrain the highly uncertain mass and angular momentum budget during the major mass transfer phase. We explore the progenitor evolution of the three best suited WR+O binaries HD 90657, HD 186943 and HD 211853, which are characterized by a WR/O mass ratio of $\sim 0.5$ and periods of $6 \ldots 10$ days. We are doing so at three different levels of approximation: predicting the massive binary evolution through simple mass loss and angular momentum loss estimates, through full binary evolution models with parametrized mass transfer efficiency, and through binary evolution models including rotation of both components and a physical model which allows to compute mass and angular momentum loss from the binary system as function of time during the mass transfer process. All three methods give consistently the same answers. Our results show that, if these systems formed through stable mass transfer, their initial periods were smaller than their current ones, which implies that mass transfer has started during the core hydrogen burning phase of the initially more massive star. Furthermore, the mass transfer in all three cases must have been highly non-conservative, with on average only $\sim 10 \%$ of the transferred mass being retained by the mass receiving star. This result gives support to our system mass and angular momentum loss model, which predicts that, in the considered systems, about $90 \%$ of the overflowing matter is expelled by the rapid rotation of the mass receiver close to the $\Omega$-limit, which is reached through the accretion of the remaining $10 \%$.
\end{abstract}

Key words. stars: binaries: close - stars: evolution - stars: fundamental parameters - stars: rotation - stars: Wolf-Rayet

\section{Introduction}

The evolution of a star in a binary system can differ significantly from that of an isolated one with the same mass and chemical composition. The physical processes that enter binary evolution are the gravitational and radiation field from the companion, as well as the centrifugal force arising from the rotation of the system. But, most important, it is the evolution of the more massive component that will influence dramatically the evolution of the system. In certain evolutionary phases, mass transfer from one star to another can occur, changing the fundamental properties of both stars as well as their future evolution.

The rotational properties of binary components may play a key role in this respect. The evolution of massive single stars can be strongly influenced by rotation (Heger \& Langer 2000; Meynet \& Maeder 2000), and evolutionary models of rotating stars are now available for many masses and metallicities. While the treatment of the rotational processes in these models is not yet in a final stage (magnetic dynamo processes are just being included Heger et al. 2004; Maeder \& Meynet 2003), they provide first ideas of what rotation can really do to a star.
Effects of rotation, as important they are in single stars, can be much stronger in the components of close binary systems: Estimates of the angular momentum gain of the accreting star in mass transferring binaries show that critical rotation may be reached quickly (Packet 1981; Langer et al. 2000; Yoon \& Langer 2004b). In order to investigate this, we need binary evolution models which include a detailed treatment of rotation in the stellar interior, as in recent single star models. However, in binaries, tidal processes as well as angular momentum and accretion need to be considered at the same time. Some first such models are now available and are discussed below.

Angular momentum accretion and the subsequent rapid rotation of the mass gainer may be essential for some of the most exciting cosmic phenomena, which may occur exclusively in binaries: type Ia supernovae, the main producers of iron and cosmic yardsticks to measure the accelerated expansion of the universe (Yoon \& Langer 2004a,b), and gamma-ray bursts from collapsars, which the most recent stellar models with rotation and magnetic fields preclude to occur in single stars (Petrovic et al. 2004; Heger et al. 2004; Woosley 2004). For both, the type Ia supernova progenitors and the gamma-ray burst 
progenitors, it is essential to understand how efficient the mass transfer process is and on which physical properties it depends. Further exciting astrophysical objects whose understanding is affected by our understanding of mass transfer comprise X-ray binaries (Chevalier \& Ilovaisky 1998) and type Ib and Ic supernovae (Podsiadlowski et al. 1992).

How much matter can stars accrete from a binary companion? As mentioned above, non-magnetic accretion, i.e. accretion via a viscous disk or via ballistic impact, transports angular momentum and can lead to a strong spin-up of the mass gaining star. For disk accretion, it appears plausible that the specific angular momentum of the accreted matter corresponds to Kepler-rotation at the stellar equator; this leads to a spin-up of the whole star to critical rotation when its initial mass is increased by about 20\% (Packet 1981). It appears possible that mass accretion continues in this situation, as viscous processes may transport angular momentum outward through the star, the boundary layer, and the accretion disk (Paczynski 1991). However, as the star is rotating very rapidly, its wind mass loss may dramatically increase (Langer 1997, 1998), which may render the mass transfer process inefficient.

Observations of massive post-mass transfer binary systems constrain this effect. Langer et al. (2003) and Langer et al. (2004) points out that there is evidence for both extremes occurring in massive close binaries, i.e. for quasi-conservative evolution as well as for highly non-conservative evolution. In the present study, we are interested in those binaries that contain a Wolf-Rayet and a main sequence $\mathrm{O}$ star. We have chosen to focus on three WN+O systems (HD 186943, HD 90657 and HD 211853) which have similar mass ratios $(\approx 0.5)$ and orbital periods (6...10 days). As clearly the two stars in these systems must have undergone a strong interaction in the past, an understanding of their progenitor evolution may be the key to constrain the mass transfer efficiency in massive binaries: which fraction of the mass leaving the primary star is accumulated by the secondary star during a mass transfer event?

Evolutionary calculations of massive close binaries were performed by various authors. General ideas about the formation of WR+O binary systems were given by Paczyński (1967), Kippenhahn et al. (1967), van den Heuvel \& Heise (1972). Vanbeveren et al. (1979) modelled the evolution of massive Case B binaries with different assumptions for mass and angular momentum loss from the binary system. Vanbeveren (1982) computed evolutionary models of massive close Case B binaries with primary masses between $20 M_{\odot}$ and $160 M_{\odot}$. He concluded that most of the WR primaries are remnants of stars initially larger than $40 M_{\odot}$ and that the accretion efficiency in these systems should be very below 0.3 in order to fit the observations. de Loore \& de Greve (1992) computed detailed models of massive Case B binary systems for initial mass ratios of 0.6 and 0.9 , assuming an accretion efficiency of 0.5 . Wellstein \& Langer (1999) and Wellstein et al. (2001) modelled massive binary systems mass range $12 \ldots 60 M_{\odot}$ assuming conservative evolution, and Wellstein (2001) presented the first rotating binary evolution models for initial masses of $\approx 15 M_{\odot}$ and initial mass ratios $q \approx 1$.

While it was realized through these models that different mass accretion may be needed to explain different observations, these efforts did not have the potential to explore the physical reasons for non-conservative evolution. I.e., there is no reason to expect that the mass transfer efficiency remains constant during the mass transfer process in a given binary system, nor that its time-averaged value is constant for whole binary populations.

It is not yet known which physical processes can expel matter from a binary system. Vanbeveren (1991) proposed that if a binary component is more massive than $\approx 40-50 M_{\odot}$ it will go through an LBV phase of enhanced mass loss, which will prevent the occurrence of RLOF. Dessart et al. (2003) investigated the possibility that radiation pressure from the secondary prevents the accretion. They found that even for moderate mass transfer rates $\left(5 \times 10^{-6} M_{\odot} \mathrm{yr}^{-1}\right)$ the wind and photon momenta which emerge from the accretion star can not alter the dynamics of the accretion stream. Here, we follow the suggestion that the effective mass accretion rate can be significantly decreased due to the spin-up of the mass receiving star (Wellstein 2001; Langer et al. 2003, 2004; Petrovic \& Langer 2004).

The remainder of this paper is organized as follows. In Sect. 2 we briefly discuss the observational data available for $\mathrm{WR}+\mathrm{O}$ binary systems. In Sect. 3 we derive estimates for the masses of both stars in $\mathrm{WR}+\mathrm{O}$ systems for given initial masses and accretion efficiencies. In Sect. 4 we present the physics used to compute our detailed evolutionary models. Non-rotating binary evolution models with an adopted constant mass accretion efficiency are presented in Sect. 5. Our rotating models in which the mass accretion efficiency is obtained selfconsistently are discussed in Sect. 6. We briefly compare our models with observations in Sect. 7. Conclusions are given in Sect. 8.

\section{Observational data}

There are about 20 observed Wolf-Rayet+O binary systems with known masses of components in the catalogue of van der Hucht (2001). We have chosen to model three spectroscopic double-lined systems: HD 186943 (WN3), HD 90657 (WN5) and GP Cep (WN6/WCE) have, since they have similar mass ratios $\left(q=M_{\mathrm{WR}} / M_{\mathrm{O}} \approx 0.5\right)$ and orbital periods (6...10 days).

$\mathrm{WN}+\mathrm{O}$ systems that also have short orbital periods are V444 Cyg, CX Cep, CQ Cep, HD 94546, HD 320102 and HD 311884. V444 Cyg has period of 4.2 days and can be result of stable mass transfer evolution, but since mass ratio of this system is $\sim 0.3$ we did not include it in this paper. Orbital periods of CX Cep and CQ Cep are very short ( $\sim 2$ days) and these systems are probably the result of a contact evolution. HD 94546 and HD 320102 are systems with very low masses of WR and $\mathrm{O}$ components $\left(4 M_{\odot}+9 M_{\odot}\right.$ and $2.3 M_{\odot}+4.1 M_{\odot}$ respectively) and HD 311884 is extremely massive $\mathrm{WR}+\mathrm{O}$ binary system $\left(51 M_{\odot}+60 M_{\odot}\right)$. Recently, an even more massive $\mathrm{WR}+\mathrm{O}$ system has been observed $83 M_{\odot}+82 M_{\odot}$ (Rauw et al. 2004; Bonanos et al. 2004).

The mass ratio of a binary system is determined from its radial velocity solution, with an error of $5-10 \%$. However, to determine the exact value of the masses of the binary components, the value of the inclination of the system has to be 
Table 1. Basic parameters of selected $\mathrm{WN}+\mathrm{O} \mathrm{SB} 2$ binaries.

\begin{tabular}{lccc}
\hline \hline WR number & WR 21 & WR 127 & WR153 ${ }^{b}$ \\
HD number & HD 90657 & HD 186943 & HD 211853 \\
spectral type & WN5+O4-6 & WN3+O9.5V & WN6/WCE+O3-6I \\
$p$ (days) & $8.2546 \pm 0.0001$ & 9.5550 & 6.6887 \\
$e$ & $0.04 \pm 0.03$ & $0.07 \pm 0.04$ & $0+0$ \\
$q$ & 0.52 & 0.47 & 0.54 \\
$a \sin i\left(R_{\odot}\right)$ & $37 \pm 3$ & $39 \pm 6$ & $>35.2$ \\
$M \sin ^{3} i\left(M_{\odot}\right)$ & 8.4 & 9.3 & \\
$i\left(^{\circ}\right)$ & $50 \pm 4$ & $55 \pm 8$ & 73 \\
$M_{\mathrm{WR}}\left(M_{\odot}\right)$ & 19 & 17 & $>6$ \\
$M_{\mathrm{O}}\left(M_{\odot}\right)$ & 37 & 36 & $>21$ \\
\hline
\end{tabular}

Notes:

${ }^{a}$ All parameters from compilation of van der Hucht (2001), unless noted otherwise.

${ }^{b}$ Demers et al. (2002).

known. Without knowledge of the inclination, only minimum masses of the components can be determined, i.e., $M \sin ^{3} i$. Massey (1981) determined the minimum mass for the WR star in HD 186943 to be $9-11 M_{\odot}$. Niemela \& Moffat (1982) determined the masses of the components of HD 90657 in the range 11-14 $M_{\odot}$ for the WN4 component and 21-28 $M_{\odot}$ for the O-type component. The masses of the WR components in HD 186943 and HD 90657 given in Table 1 have been determined by Lamontagne et al. (1996) on the basis of improved values for the inclination of these systems. Demers et al. (2002) determined minimum masses of the components of the system GP Cep. Previously, Lamontagne et al. (1996) suggested values of $M_{\mathrm{WR}}=15 M_{\odot}$ and $M_{\mathrm{O}}=27 M_{\odot}$ for this system.

There is no obvious hydrogen contribution in the WR spectrum in any of these systems (Massey 1981; Niemela \& Moffat 1982). Massey (1981) showed that hydrogen absorption lines are fairly broad in the spectrum of HD 186943, equivalent to $v \sin i \simeq 250 \mathrm{~km} \mathrm{~s}^{-1}$, thus the O-type star is rotating much faster than synchronously.

Beside the fact that the binary system GP Cep has a similar mass ratio and period as the other two systems, it has some very different properties as well. The spectral type of the WR component in GP Cep is a combination of WN and WC (WN6/WCE Demers et al. 2002). Also, Massey (1981) showed that, next to the main period of $\sim 6.69$ days of the binary system GP Cep, radial velocities of absorption lines vary also with a period of 3.4698 days. He proposed that GP Cep is a quadruple system, consisting of two pairs of stars, $\mathrm{WR}+\mathrm{O}$ and $\mathrm{O}+\mathrm{O}$. Panov \& Seggewiss (1990) suggested that in both pairs one component is a WR star. However, Demers et al. (2002) showed that there is only one WR star in this quadruple system.

\section{The simple approach}

If the initial binary system is very close (an initial period is of the order of few days), RLOF occurs while the primary is still in the core hydrogen burning phase and Case A mass transfer takes place (fast and slow phase). When the primary expands due to shell hydrogen burning, it fills its Roche lobe and Case AB mass transfer starts. During this mass transfer the primary star loses the major part of its hydrogen envelope. After Case AB mass transfer, the primary is a helium core burning Wolf-Rayet star. During all this time, the secondary is still a main sequence star, but with an increased mass due to mass transfer. When the initial binary period is of the order of one to few weeks, the primary fills its Roche lobe for the first time during shell hydrogen burning and Case B mass transfer takes place. The primary loses most of its hydrogen envelope, becomes a WR star and the secondary is an O star with an increased mass. Case $C$ mass transfer occurs when initial period is of the order of years. The primary fills its Roche lobe during helium shell burning and mass transfer takes place on the dynamical time scale. This scenario is not likely for chosen systems, since some of the secondary stars in WR+O systems have been observed to rotate faster than synchronously. This means that they have accreted some matter which increased their spin angular momentum.

We constructed a simple method to quickly estimate the post-mass transfer parameters for a large number of binary systems for a given accretion efficiency $\beta$. This allows us to narrow the space of possible initial parameters (primary mass, secondary mass and orbital period) that allows the evolution into a specific observed WR+O systems.

We considered binary systems with initial primary masses $M_{1, \text { in }}=25 \ldots 100 M_{\odot}$ and secondaries masses $M_{2, \text { in }}=$ $25 / 1.7 \ldots 100 M_{\odot}$ with an initial period of 3 days. We assumed that the primary is transferring matter to the secondary until it reaches the mass of its initial helium core (Eq. (1)).

Matter that is not accreted on the secondary leaves the system with the specific angular momentum which corresponds to the secondary's orbital angular momentum (King et al. 2001), which is consistent with our approach for mass loss from the binary system (cf. Sect. 4). Stellar wind mass loss is neglected.

More massive initial primaries produce more massive WR stars (helium cores) in general, but if the star is in a binary system that goes through mass transfer during hydrogen core burning of the primary (Case A), this depends also on other parameters:

- If the initial period is longer, mass transfer starts later in the primary evolution and the initial helium core of the primary is more massive.

- If the initial mass ratio is further from unity, the mass transfer rate from the primary reaches higher values and the initial helium core mass is smaller.

We initially want to restrict ourselves to systems that undergo stable mass transfer, i.e. avoid contact situations. Wellstein et al. (2001) found that the limiting initial mass ratio for conservative Case A binary system is $M_{1 \text {,in }} / M_{2 \text {,in }} \sim 1.55$ and for conservative Case B systems $\sim 1.25$. Since we allow nonconservative evolution, we consider initial mass ratio $q \leq 1.7$ for Case A and $q \leq 1.4$ for Case B. The observed WR+O systems (HD 186943, HD 90657 and HD 211853) all have very short orbital periods, between 6 and 10 days. Since, the net effect of the Case A+Case AB, or Case B is a widening of the orbit (if there is no contact), we have to assume that the initial periods need to be shorter than, or approximatively equal 
to the observed ones. We adopted a minimum initial orbital period of 3 days to avoid that the primary fills its Roche lobe on the ZAMS.

We estimated the minimum initial helium core masses, which are obtained by the earliest Case A systems, for systems with a mass ratio of $M_{1, \text { in }} / M_{2 \text {,in }}=1.7$ and an initial period of 3 days $\left(M_{1 \text {,in }} \gtrsim 41 M_{\odot}\right)$ from the detailed evolutionary models shown later in this paper (Sect. 5):

$M_{\mathrm{WR}, \text { in }}=0.24 * M_{1, \text { in }}+0.27$.

In this linear approximation, we neglected the influence of the initial mass ratio on the initial WR mass. It is shown in Sect. 5.2 that this dependence becomes important only for initial mass ratios above $q \simeq 2$.

For Case B binaries, the initial WR mass does not depend on the initial period and the initial mass ratio of the system, since during core hydrogen burning, the primary evolves as a single star, without any interaction with the secondary. We estimated the relation between initial main sequence mass and initial WR mass as a linear fit from the Case B binary systems with initial primaries $M_{1 \text {,in }} \gtrsim 18 M_{\odot}$ (Wellstein \& Langer 1999):

$M_{\mathrm{WR}, \text { in }}=0.53 * M_{1, \text { in }}-4.92$.

The minimum initial period for a system to evolve through Case B mass transfer depends on the initial primary mass and the mass ratio. We can estimate, based on the radii of the primaries at the end of the main sequence evolution, what would be the initial orbital separation necessary to avoid the primary filling its Roche lobe before shell hydrogen burning. From Kepler's law follows that the orbital separation is proportional to the mass ratio $a \sim q^{-1 / 3}$ and the initial primary mass $a \sim M_{1}{ }^{1 / 3}$. Since $q \sim 1$ we can neglect this dependence and estimate the initial period for which the radius of the primary at the end of MS is equal to its Roche radius. For this estimate we do not take into account stellar wind that will widen the orbit and decrease the masses. We conclude that the Case B limiting initial orbital period for $40 M_{\odot}$ is $\sim 10$ days, for $45 M_{\odot} \sim 15$ days and for $75 M_{\odot} \sim 30$ days. Since the result of stable Case B mass transfer is widening of the orbit, it follows that (stable mass transfer) Case B binary systems can not be progenitors of the observed systems HD 186943, HD 90657 and HD 211853 whose orbital periods are shorter than 10 days. However, WR star masses resulting from Case B evolution are practically the same as those from very late Case A evolution, which is still considered in our analysis.

We calculate binary systems for early Case $\mathrm{A}$ ( $p_{\text {in }}=3$ days) and for late Case $\mathrm{A}\left(p_{\text {in }} \approx p_{\text {limit }}\right)$.

The results are shown in Fig. 1 for four different accretion efficiencies $(\beta=0.0,0.1,0.5,1.0$ respectively) for early Case $\mathrm{A}$ evolution ( $p=3$ days). Figure 2 shows the results for early Case A systems ( $p=3$ days) for all values of $\beta=0 \ldots 1$ and for Case $\mathrm{B} /$ late Case $\mathrm{A}$, also for all $\beta$.

We notice from Fig. 1, that when the assumed $\beta$ is larger, the resulting $\mathrm{WR}+\mathrm{O}$ systems lie further from the line defined by $q=0.5$. The reason is clear: if the accretion efficiency is higher, the secondary will become more massive while the initial mass of the WR star stays the same. Conservative evolution

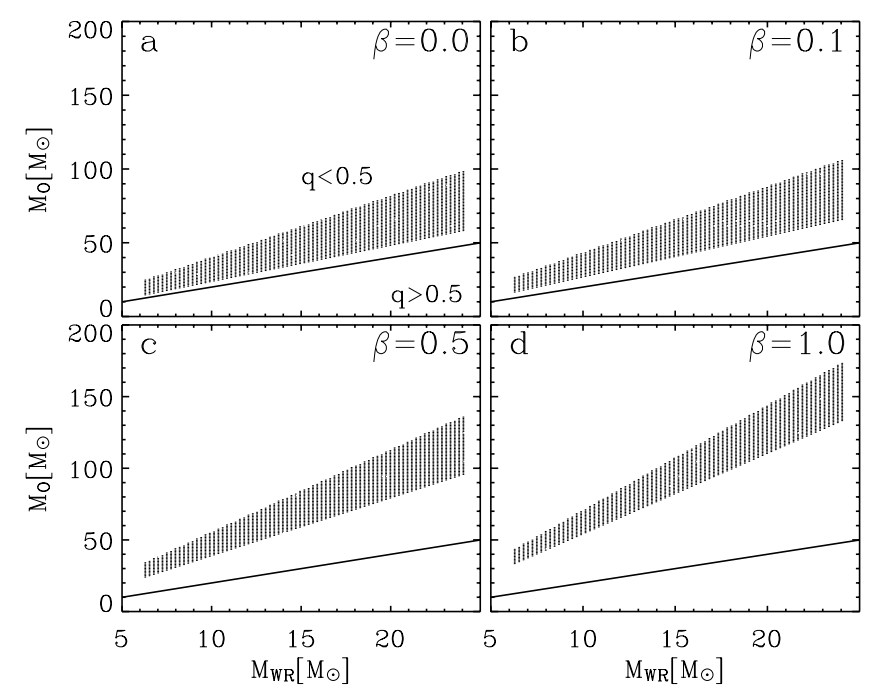

Fig. 1. Masses of both components of post-Case A mass transfer $\mathrm{WR}+\mathrm{O}$ binary systems resulting from our simple approach, for initial primary masses in the range $25 \ldots 100 M_{\odot}$ and an initial period of $p_{\text {in }}=$ 3 days, for four different assumed accretion efficiencies $\beta(\mathbf{a})-\mathbf{d})$ ). The solid line represents a mass ratio of $q=M_{\mathrm{WR}} / M_{\mathrm{O}}=0.5$. For an increasing $\beta$, the $\mathrm{O}$ stars in $\mathrm{WR}+\mathrm{O}$ systems become more massive and the WR/O-mass ratio decreases.

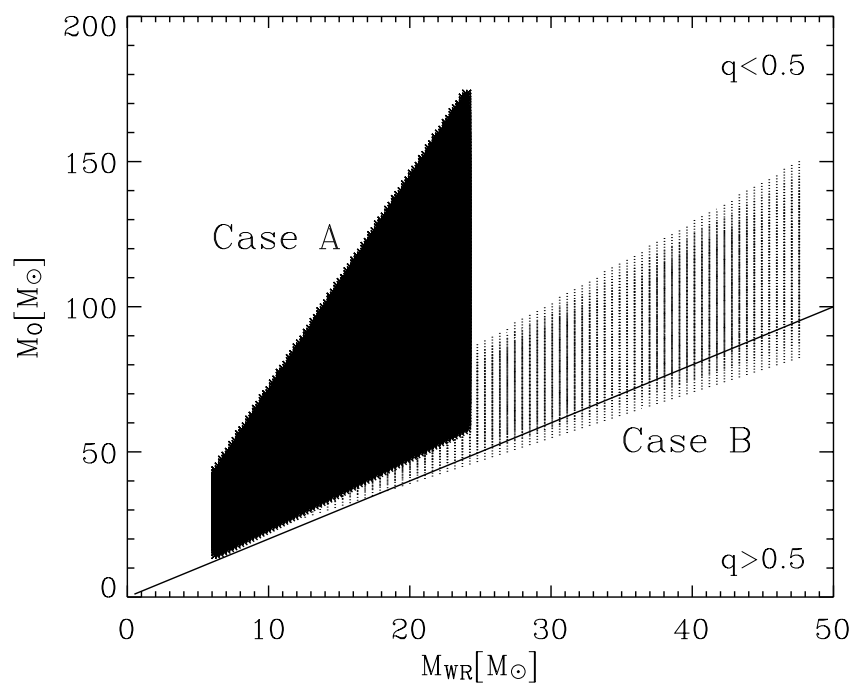

Fig. 2. Masses of both components of post-Case A mass transfer $\mathrm{WR}+\mathrm{O}$ binary systems resulting from our simple approach, for initial primary masses in the range $25 \ldots 100 M_{\odot}$ and for early ( $p_{\text {in }}=3$ days) Case A and late Case A respective Case B evolution. The assumed accretion efficiency is $\beta=0 \ldots 1$. The solid line represents a mass ratio of $q=M_{\mathrm{WR}} / M_{\mathrm{O}}=0.5$.

(Fig. 1d) produces $\mathrm{WR}+\mathrm{O}$ systems that have small mass ratios, $q=1 / 5 \ldots 1 / 6$.

We conclude that if the considered three observed $\mathrm{WR}+\mathrm{O}$ binary systems evolved through a stable mass transfer, a large amount of matter must have left the system. On the other hand, since some of the secondary stars in WR+O binaries have been observed to rotate faster than synchronously (Massey 1981; Underhill et al. 1988), a certain amount of accretion may be required. 
Figure 2 shows the resulting $\mathrm{WR}+\mathrm{O}$ masses in Case $\mathrm{A}$ and Case B (latest Case A) for accretion efficiency $\beta=0 \ldots 1$. If the primary star does not lose mass in a mass transfer during core hydrogen burning ( $\left.p_{\text {in }} \geq p_{\text {limit }}\right)$, it will form a more massive WR star, as we already explained. There will be less mass to transfer from the primary to the secondary, and for fixed $\beta$ the corresponding $\mathrm{O}$ star will become less massive. However, since the observed periods of HD 186943, HD 90657 and HD 211853 are shorter than 10 days and Case $\mathrm{A}+\mathrm{Case} \mathrm{AB}$ widens the binary orbit, the initial orbital period should be shorter than observed, so we can conclude roughly that $p_{\text {in }}$ is between 3 and 10 days.

The orbital period of $\mathrm{WR}+\mathrm{O}$ systems depends on their initial orbital period, their initial mass ratio and on the parameter $\beta$. If the initial period increases and there is no contact during the evolution, the orbital period in the $\mathrm{WR}+\mathrm{O}$ stage will also increase. However, the orbital period of $\mathrm{WR}+\mathrm{O}$ systems will be shorter if the initial mass ratio is larger. If the initial masses are very similar, the primary will become less massive than the secondary very early during the mass transfer, and afterward matter is transfered from the less to the more massive star, which results in a widening of the orbit. Conversely, the final period is shorter for a larger difference in initial masses in the binary system.

We can draw the following conclusions:

- The accretion efficiency during the major mass transfer phase in the progenitor evolution of the three observed $\mathrm{WR}+\mathrm{O}$ binaries is small, i.e. $\beta=0 \ldots 0.1$, as for larger $\beta$ the $\mathrm{O}$ stars during the $\mathrm{WR}+\mathrm{O}$ phase are more massive and the WR/O-mass rations smaller than observed. However, we note that it is unlikely that the secondaries did not accrete at all $(\beta=0)$, since some $\mathrm{O}$ stars are found to rotate faster than synchronously.

- The initial orbital period needs to be larger than $\sim 3$ days, to avoid contact at the beginning of hydrogen burning.

- The initial orbital period should be larger than $\sim 3$ days, in order to obtain massive enough WR stars.

- The initial orbital periods should be shorter than the observed orbital periods in the three $\mathrm{WR}+\mathrm{O}$ systems, i.e. shorter than $\sim 10$ days. This excludes Case B mass transfer.

- While the initial mass ratio $M_{1, \text { in }} / M_{2, \text { in }}$ should not be too far from unity so contact is avoided, it should be close to the contact limit, since this leads to the shortest orbital periods and largest $\mathrm{WR} / \mathrm{O}$ mass ratios in $\mathrm{WR}+\mathrm{O}$ systems, as needed for the three observed systems.

\section{Numerical code and physical assumptions}

We showed in Sect. 3 that we can roughly estimate the parameters of the progenitor systems of observed $\mathrm{WR}+\mathrm{O}$ binaries HD 186943, HD 90657 and HD 211853. However, detailed numerical models are required in order to verify that the assumption of contact-free evolution can in fact be justified. And finally, we want to check whether the required mass and angular momentum loss can be reproduced by our detailed selfconsistent approach.
We are using a binary evolutionary code which was originally developed by Braun (1998) on the basis of an implicit hydrodynamic stellar evolution code for single stars (Langer 1991, 1998). It calculates simultaneous evolution of the two stellar components of a binary system in a circular orbit and the mass transfer within the Roche approximation (Kopal 1978). Mass loss from the Roche lobe filling component through the first Lagrangian point is given by Ritter (1988) as:

$\dot{M}=\dot{M}_{0} \exp \left(R-R_{1}\right) / H_{\mathrm{p}}$

with $\dot{M}_{0}=\rho v_{\mathrm{s}} Q / \sqrt{e}$, where $H_{\mathrm{p}}$ is the photospheric pressure scale height, $\rho$ is the density, $v_{\mathrm{s}}$ the velocity of sound and $Q$ the effective cross-section of the stream through the first Lagrangian point according to Meyer \& Meyer-Hofmeister (1983).

Stellar wind mass loss for $\mathrm{O}$ stars on the main sequence is calculated according to Kudritzki et al. (1989). For hydrogenpoor stars $\left(X_{\mathrm{s}}<0.4\right)$ we assume mass loss based on the empirical mass loss rates for Wolf-Rayet stars derived by Hamann et al. (1995):

$\log \left(\dot{M}_{\mathrm{WR}} / M_{\odot} \mathrm{yr}^{-1}\right)=-11.95+1.5 \log L / L_{\odot}-2.85 X_{\mathrm{s}}$.

Since Hamann \& Koesterke (1998) suggested that these mass loss rates may be overestimated, we calculated evolutionary models with mass loss rate given by Eq. (4) multiplied by factors $1 / 2,1 / 3$ and $1 / 6$.

The treatment of a convection and a semiconvection which is applied here is described in Langer (1991) and Braun \& Langer (1995). Changes in chemical composition are computed using a nuclear network including pp chains, the CNO-cycle, and the major helium, carbon, neon and oxygen burning reactions. More details are given in Wellstein \& Langer (1999) and Wellstein et al. (2001). We use the OPAL Rosseland-mean opacities of (Iglesias \& Rogers 1996). For all models, a metallicity of $Z=0.02$ is adopted. The abundance ratios of the isotopes for a given element are chosen to have the solar meteoritic abundance ratios according to Grevesse \& Noels (1993). The change of the orbital period (orbital angular momentum loss) due to the mass transfer and stellar wind mass loss is computed according to Podsiadlowski et al. (1992), with the specific angular momentum of the stellar wind material calculated by Brookshaw \& Tavani (1993).

The influence of the centrifugal force in the rotating models is implemented according to Kippenhahn \& Thomas (1970). The stellar spin vectors are assumed to be perpendicular to the orbital plane. Synchronization due to tidal spin-orbit coupling is included with a time scale given by Zahn (1977). Rotationally enhanced mass loss is included as follows:

$\dot{M} / \dot{M}\left(v_{\mathrm{rot}}=0\right)=1 /(1-\Omega)^{\xi}$,

where $\xi=0.43, \Omega=v_{\text {rot }} / v_{\text {crit }}$ and $v_{\text {crit }}^{2}=G M(1-\Gamma) / R$ with $\Gamma=L / L_{\mathrm{Edd}}=\kappa L /(4 \pi c G M)$ is Eddington factor, $G$ is gravitational constant, $M$ is mass, $R$ radius, $\kappa$ opacity, $v_{\text {rot }}$ rotating velocity and $v_{\text {crit }}$ critical rotational velocity (Langer 1998).

When the star approaches $\Omega=1$, the mass loss rate is increased according to the previous equation. However, mass loss also causes a spin-down of the star and equilibrium mass loss 
rate $\Omega_{\mathrm{eq}}$ results (Langer 1998). If $\Omega>\Omega_{\mathrm{eq}}$, the corresponding angular momentum loss is so large that the star evolves away from the $\Omega$-limit.

The transport of angular momentum through the stellar interiour is formulated as a diffusive process:

$$
\left(\frac{\partial \omega}{\partial t}\right)_{m}=\frac{1}{i}\left(\frac{\partial}{\partial m}\right)_{t}\left[\left(4 \pi r^{2} \rho\right)^{2} i v\left(\frac{\partial \omega}{\partial m}\right)_{t}\right]-\frac{2 w}{r}\left(\frac{\partial r}{\partial t}\right)_{m} \frac{1}{2} \frac{\mathrm{d} \ln i}{\mathrm{~d} \ln r}
$$

where $v$ is the turbulent viscosity and $i$ is the specific angular momentum of a shell at mass coordinate $m$.

The specific angular momentum of the accreted matter is determined by integrating the equation of motion of a test particle in the Roche potential in case the accretion stream impacts directly on the secondary star, and is assumed Keplerian otherwise Wellstein (2001). Rotationally induced mixing processes and angular momentum transport through stellar interior are described by Heger et al. (2000). Magnetic fields generated due to differential rotation in the stellar interior (Spruit 2002) are not included here (however, see Petrovic et al. 2004).

We calculated the evolution of the binary systems in detail until Case $\mathrm{AB}$ mass transfer starts. Then we estimated the outcome of this mass transfer by assuming that it ends when WR star has $\sim 5 \%$ of the hydrogen left at the surface. For this purpose we calculate the Kelvin-Helmholtz time scale of the primary:

$t_{\mathrm{KH}}=2 \times 10^{7} M_{1}^{2} /\left(L_{1} R_{11}\right) \mathrm{yr}$

where $M_{1}, L_{1}$ and $R_{11}$ are mass, luminosity and Roche radius (in Solar units) of the primary star at the onset of Case AB mass transfer. The mass transfer rate is then assumed as:

$\dot{M}_{\mathrm{tr}}=\left(M_{1}-M_{\mathrm{WR}, \text { in }}\right) / t_{\mathrm{KH}}$

where $M_{\mathrm{WR}, \text { in }}$ is the mass of the WR star that has a hydrogen surface abundance of 5\%; all quantities are taken at the beginning of the mass transfer. We calculate the change of the orbital period orbit using constant value of $\beta=0.1$ for non-rotating and $\beta=0.0$ for rotating models (Wellstein 2001). Matter that is not retained by the secondary is assumed to leave the system with a specific angular momentum which corresponds to the secondary's orbital angular momentum (King et al. 2001).

\section{Non-rotating models}

We concluded in Sect. 3 that massive $\mathrm{O}+\mathrm{O}$ binaries can result in WR+O systems similar to observed the (HD 186943, HD 90657 and HD 211853) if accretion efficiency $\beta$ is low. Since some $\mathrm{O}$ stars in $\mathrm{WR}+\mathrm{O}$ binaries have been observed to rotate faster than synchronously, we concluded that $\beta>0.0$ and assumed a constant value of $\beta=0.1$ in our detailed evolutionary models. We already mentioned that the orbital periods of the observed systems are between 6 and 10 days. Since the net effect of Case $A+C$ ase $A B$ mass transfer is a widening of the orbit, the initial periods should be shorter than the observed ones, so we modelled binary systems with initial orbital periods of 3 and 6 days.

We chose initial primary masses to be in the range $41 \ldots 75 M_{\odot}$. The masses of the secondaries are chosen so that the initial mass ratio $\left(M_{1, \text { in }} / M_{2, \text { in }}\right)$ is $q \approx 1.7-2.0$. An initial mass ratio of $\approx 1.55$ is estimated to be the limiting value for the occurrence of contact between the components in Case A systems by Wellstein et al. (2001) for conservative mass transfer. Contact occurs when the accretion time scale of the secondary $\left(\dot{M}_{2, \text { acc }} / M_{2}\right)$ is much longer than the thermal (KelvinHelmholtz) time scale of the primary $\left(\dot{M}=M_{1} / t_{\mathrm{KH}}\right)$, so the secondary expands and fills its Roche lobe. In our models, only $10 \%$ of matter lost by the primary is accreted on the secondary star, so it reaches hydrostatic equilibrium faster and expands less than in the case of larger $\beta$. This is the reason why we adopted a weaker condition for contact formation and calculate models with mass ratios $q \approx 1.7$...2.0.

All modelled systems (except the ones that enter contact) go through Case A and Case AB mass transfer. Details of the evolution of all calculated binary systems are given in Table 2. We discuss the details of the binary evolution taking the system number 11 as an example. Figure 3 shows the evolutionary tracks of the primary and the secondary in the HR diagram until the onset of Case $\mathrm{AB}$ mass transfer. This system begins its evolution with the initial parameters $M_{1, \text { in }}=56 M_{\odot}$, $M_{2, \text { in }}=33 M_{\odot}, p_{\text {in }}=6$ days. Both stars are core hydrogen burning stars (dashed line, Fig. 3), but since the primary is more massive, it evolves faster and fills its Roche lobe, so the system enters Case A mass transfer (solid line, Fig. 3) $\sim 5.6 \times 10^{6}$ years after the beginning of core hydrogen burning. The first phase of Case $\mathrm{A}$ is fast process and takes place on the Kelvin-Helmholtz (thermal) time scale $\left(\sim 3.1 \times 10^{4}\right.$ years). The primary loses matter quickly and continuously with a high mass transfer rate $\left(\dot{M}_{\mathrm{tr}}^{\max } \sim 3.1 \times 10^{-3} M_{\odot} \mathrm{yr}^{-1}\right)$. In order to retain hydrostatic equilibrium, the envelope expands, which requires energy and causes a decrease in luminosity (Fig. 3). At the same time the secondary is accreting matter and is expanding. Due to this, its luminosity increases and the effective temperature decreases (Fig. 3). During fast phase of Case A mass transfer the primary loses $\sim 19 M_{\odot}$ and the secondary accretes $1 / 10$ of that matter. After the fast process of mass transfer, the primary is still burning hydrogen in its core and is still expanding, so slow phase of Case A mass transfer takes place on a nuclear time scale $\left(0.46 \times 10^{6}\right.$ years $)$ with a mass transfer rate of $\dot{M}_{\text {tr }} \sim 10^{-6} M_{\odot} \mathrm{yr}^{-1}$. After this, the primary is the less massive star, with decreased hydrogen surface abundance. Stellar wind mass loss of the primary increases when its surface becomes hydrogen poor $\left(X_{\mathrm{s}}<0.4\right)$. At the end of core hydrogen burning the primary contracts (effective temperature increases) and thus RLOF stops (Fig. 3 dotted line). When the primary starts shell hydrogen burning it expands (dash-dotted line, Fig. 3), fills its Roche lobe and Case AB mass transfer starts.

Figures 4 and 5 show the evolution of the interior of the primary and the secondary until Case $\mathrm{AB}$ mass transfer. The primary loses huge amounts of matter during fast Case A mass transfer and its convective core becomes less than a half of its original mass. At the same time, the secondary accretes matter from the primary and the heavier elements are being relocated by thermohaline mixing. In Figs. 6 and 7 we see the mass transfer rate and the surface abundances of hydrogen, carbon, nitrogen and oxygen. 


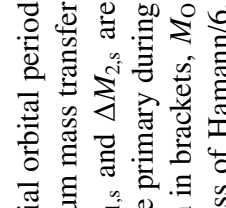

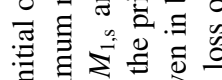

稀

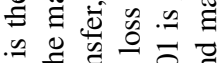

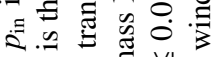

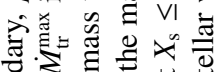

过《.

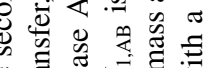

Е

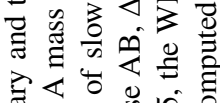

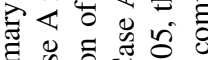

急记

녕

范 卷

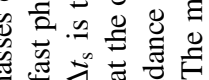

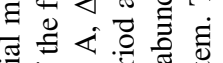

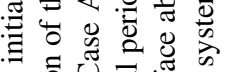

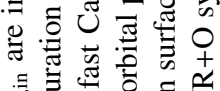

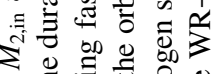

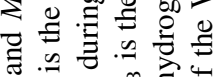

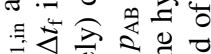

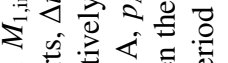

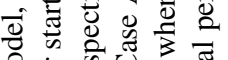

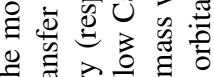

ङ 焉

पे

过纯

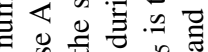

娄

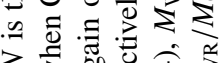

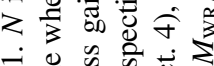

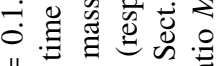

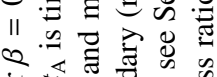

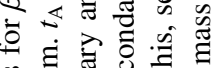

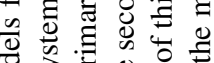

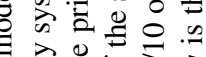

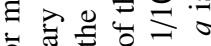

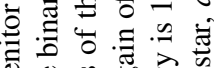

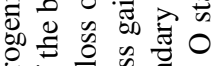

년

ㅇํㅀ

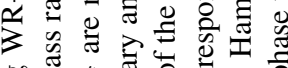

on

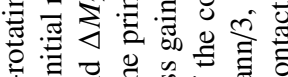

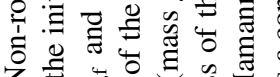

$z$
$i$
0

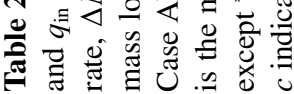

\begin{tabular}{|c|c|c|c|c|c|c|c|c|c|c|c|c|c|c|c|}
\hline 2 & $\nabla$ & 1 & 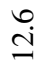 & $\stackrel{n}{\mathfrak{n}}$ & $\stackrel{n}{m}$ & $\frac{n}{\sim}$ & $\begin{array}{l}\stackrel{0}{0} \\
\stackrel{0}{ }\end{array}$ & $\stackrel{\infty}{\stackrel{i}{ }}$ & 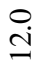 & $\begin{array}{l}\infty \\
\sigma\end{array}$ & $\stackrel{\check{n}}{\ddot{n}}$ & $\begin{array}{l}\infty \\
\dot{\Omega}\end{array}$ & $\overrightarrow{\mathrm{I}}$ & 1 & 1 \\
\hline$\sigma$ & & 1 & กี & $\tilde{\dddot{m}}$ & $\stackrel{\infty}{\tilde{c}}$ & $\stackrel{0}{+}$ & $\stackrel{n}{\tilde{c}}$ & $\stackrel{m}{0}$ & ले & 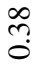 & $\tilde{n}$ & $\tilde{n}$ & $\stackrel{n}{n}$ & 1 & 1 \\
\hline$\Sigma$ & $\Sigma^{\circ}$ & 1 & הֶ. & ֶָ) & $\begin{array}{l}\vec{d} \\
\text { d. }\end{array}$ & ?్ֶలి & $\vec{a}$ & $\frac{\infty}{\dot{m}}$ & $\begin{array}{l}\text { ते } \\
\text {. }\end{array}$ & $\stackrel{\forall}{\ddot{n}}$ & $\vec{m}$ & $\stackrel{q}{\dot{m}}$ & $\begin{array}{l}n \\
\stackrel{n}{m}\end{array}$ & 1 & 1 \\
\hline 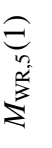 & $\Sigma^{\ominus}$ & 1 & $\begin{array}{l}\underset{\overbrace{}}{\Xi} \\
\underset{\infty}{\Xi} \\
\stackrel{\Xi}{=}\end{array}$ & 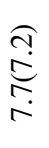 & $\begin{array}{l}\widehat{\overbrace{}} \\
\stackrel{0}{0} \\
0 \\
0\end{array}$ & 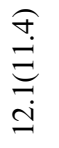 & $\begin{array}{l}\hat{\infty} \\
\stackrel{0}{0} \\
\stackrel{m}{0}\end{array}$ & 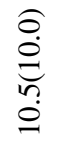 & 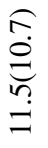 & 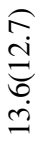 & $\begin{array}{l}\widetilde{n} \\
\stackrel{2}{\Xi} \\
\infty \\
\infty \\
-\end{array}$ & 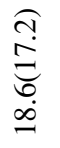 & $\begin{array}{l}\underset{f}{\sigma} \\
\underset{ٍ}{\infty} \\
\stackrel{\infty}{0}\end{array}$ & 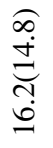 & 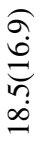 \\
\hline$\sum_{4}^{\infty}$ & $\Sigma^{\circ}$ & 1 & $\vec{r}$ & $\underset{\infty}{\infty}$ & $\stackrel{n}{\sigma}$ & $\stackrel{n}{n}$ & $\stackrel{n}{a}$ & $\stackrel{0}{\stackrel{0}{a}}$ & $\begin{array}{l}\bar{\infty} \\
\infty \\
\infty\end{array}$ & $\underset{⿱}{\stackrel{\Delta}{r}}$ & $\stackrel{\infty}{a}$ & $\stackrel{n}{\frac{n}{n}}$ & $\stackrel{n}{m}$ & 1 & 1 \\
\hline$\sum_{2}^{\infty}$ & $\nabla$ & 1 & in & 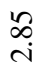 & $\begin{array}{c}\infty \\
\dot{\infty}\end{array}$ & $\underset{\infty}{\sigma}$ & $\stackrel{\infty}{\stackrel{\infty}{\sim}}$ & $\begin{array}{l}\text { त̂ } \\
\text { in }\end{array}$ & $\begin{array}{l}\infty \\
\infty \\
\dot{\rho}\end{array}$ & $\stackrel{\delta}{\dot{r}}$ & $\stackrel{ }{\stackrel{R}{r}}$ & $\bar{a}$ & $\begin{array}{l}\infty \\
\infty \\
\infty\end{array}$ & 1 & 1 \\
\hline 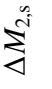 & $\Sigma^{\circ}$ & 1 & $\stackrel{8}{\circ}$ & 1 & तึ & $\stackrel{8}{\circ}$ & $\stackrel{5}{0}$ & $\stackrel{\infty}{\circ}$ & $\stackrel{n}{\tilde{c}}$ & 守 & $\underset{0}{0}$ & $\stackrel{8}{0}$ & ộ & 1 & 1 \\
\hline$\sum^{n}$ & $\Sigma^{\circ}$ & 1 & à & 1 & $\frac{m}{n}$ & 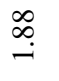 & $\begin{array}{l}\infty \\
\infty \\
i \\
i\end{array}$ & $\underset{\sigma}{\mathbb{b}}$ & 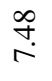 & $\begin{array}{l}\stackrel{8}{0} \\
\stackrel{2}{2}\end{array}$ & $\stackrel{尺}{\stackrel{f}{f}}$ & $\stackrel{\beta}{r}$ & $\stackrel{q}{r}$ & 1 & 1 \\
\hline$\ddot{z}$ & $\mid \begin{array}{l}5 \\
0 \\
0\end{array}$ & 1 & के & 1 & $\stackrel{\vec{n}}{-}$ & İ. & $\stackrel{\vec{n}}{-}$ & $\stackrel{\vec{n}}{-}$ & $\tilde{n}$ & $\stackrel{\infty}{\stackrel{\infty}{-}}$ & $\stackrel{8}{:}$ & $\stackrel{0}{\stackrel{0}{0}}$ & $\stackrel{q}{\stackrel{0}{0}}$ & 0 & $u$ \\
\hline$\sum_{4}^{4}$ & $\Sigma^{\circ}$ & 1 & $\stackrel{\infty}{\infty}$ & $\overrightarrow{\vec{i}}$ & $\stackrel{\vec{n}}{\rightarrow}$ & $\stackrel{\Omega}{\stackrel{I}{-}}$ & $\hat{n}$ & 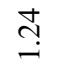 & $\stackrel{n}{n}$ & $\stackrel{?}{\stackrel{2}{I}}$ & 9 & $\stackrel{9}{-}$ & $\stackrel{9}{-}$ & $\stackrel{\infty}{-\infty}$ & $\stackrel{શ}{\stackrel{2}{-}}$ \\
\hline$\sum_{8}^{3}$ & $\AA^{\ominus}$ & 1 & $\begin{array}{l}\mathcal{N} \\
\infty \\
\infty\end{array}$ & $\stackrel{m}{\dot{\lambda}}$ & $\stackrel{\infty}{n}$ & $\stackrel{\bar{m}}{=}$ & $\begin{array}{l}\tilde{\infty} \\
\stackrel{\Omega}{a}\end{array}$ & $\begin{array}{l}\underset{B}{B} \\
\text { I }\end{array}$ & $\begin{array}{l}\vec{f} \\
\stackrel{a}{a}\end{array}$ & 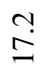 & $\begin{array}{l}\stackrel{\mathfrak{m}}{2} \\
\stackrel{2}{2}\end{array}$ & $\begin{array}{l}\stackrel{\sim}{2} \\
\stackrel{2}{2}\end{array}$ & $\stackrel{\check{m}}{\varrho}$ & $\begin{array}{l}\vec{\infty} \\
\infty \\
\infty\end{array}$ & $\begin{array}{l}\hat{i n} \\
\infty \\
\infty\end{array}$ \\
\hline 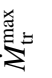 & 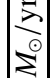 & 1 & $\stackrel{+}{i}$ & $\stackrel{\infty}{\infty}$ & $\underset{r}{\dot{r}}$ & $\ddot{n}$ & 9 & $\exists$ & $\stackrel{m}{m}$ & $\ddot{r}$ & $\bar{m}$ & $\bar{m}$ & $\vec{m}$ & $\stackrel{r}{\forall}$ & $\bar{m}$ \\
\hline $\bar{y}$ & 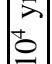 & u & $\overrightarrow{\dot{\omega}}$ & ָָ & $\bar{m}$ & $\stackrel{?}{\rightarrow}$ & $\begin{array}{l}\infty \\
\dot{n}\end{array}$ & $\hat{\sigma}$ & $\stackrel{\vec{r}}{\dot{m}}$ & $\dot{i n}$ & $\stackrel{\infty}{\dot{n}}$ & $\begin{array}{l}\infty \\
\dot{n}\end{array}$ & $\begin{array}{l}\infty \\
\dot{n}\end{array}$ & $\stackrel{\sim}{\sim}$ & $\stackrel{\mathcal{Y}}{\mathfrak{Y}}$ \\
\hline$\leqslant$ & $\mid \begin{array}{l}5 \\
0 \\
0\end{array}$ & $\stackrel{\infty}{i}$ & $\stackrel{\circ}{\sim}$ & $\stackrel{\infty}{i}$ & $\stackrel{\infty}{i}$ & $\stackrel{\bullet}{\dot{r}}$ & $\stackrel{n}{\stackrel{n}{i}}$ & $\hat{i}$ & $\stackrel{n}{i}$ & 9 & 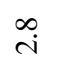 & $\stackrel{\infty}{i}$ & $\stackrel{\infty}{i}$ & $\stackrel{\circ}{\rightarrow}$ & $\stackrel{?}{9}$ \\
\hline$\Xi$ & & $\stackrel{n}{i}$ & 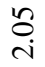 & $\underset{i}{\stackrel{i}{i}}$ & $\underset{I}{\mathrm{I}}$ & $\stackrel{ }{I}$ & $\stackrel{\tilde{n}}{n}$ & $\stackrel{m}{m}$ & $\stackrel{్}{\sigma}$ & $\stackrel{?}{i}$ & $\stackrel{?}{I}$ & $\stackrel{?}{\stackrel{-}{*}}$ & $\stackrel{?}{I}$ & $\stackrel{゚}{\stackrel{2}{*}}$ & \\
\hline 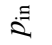 & -0 & $m$ & 0 & $m$ & $m$ & 0 & $n$ & $m$ & $m$ & $m$ & 0 & 0 & 0 & $m$ & $m$ \\
\hline 里 & $\Sigma^{\circ}$ & ¿ิ & $\stackrel{\lambda}{ }$ & 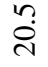 & $\stackrel{+}{\mathrm{d}}$ & $\stackrel{\sim}{\sim}$ & $\bar{\lambda}$ & \& & $\grave{\lambda}$ & $\tilde{m}$ & $\tilde{m}$ & m & $\ddot{m}$ & $\hat{m}$ & r \\
\hline$\sum$ & $\Sigma^{\circ}$ & $\bar{F}$ & $\exists$ & F & $\vec{F}$ & $\vec{F}$ & $\vec{F}$ & $\bar{\forall}$ & ケ & in & in & in & in & $\approx$ & 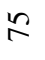 \\
\hline$z$ & & $\bar{z}$ & z & $\tilde{z}$ & 艺 & z & $z$ & $\bar{z}$ & $\stackrel{z}{Z}^{\infty}$ & zे & $\stackrel{\circ}{z}$ & $\stackrel{*}{\bar{z}}$ & $\stackrel{\stackrel{u}{Z}}{\check{z}}$ & $\frac{m}{z}$ & $\vec{z}$ \\
\hline
\end{tabular}
I 


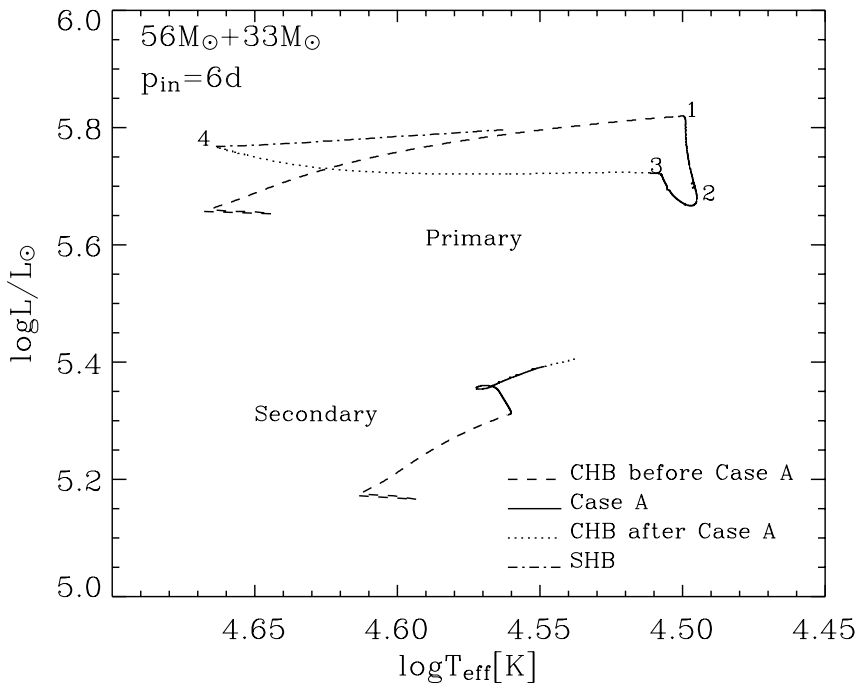

Fig. 3. HR diagram of the initial system $M_{1, \text { in }}=56 M_{\odot}, M_{2, \text { in }}=33 M_{\odot}$, $p_{\text {in }}=6$ days. Both stars are core hydrogen burning (dashed line) until Case A mass transfer starts (solid line). The primary is losing mass and its luminosity and effective temperature decrease. At the same time the secondary is accreting matter and expanding, becoming more luminous and cooler. After Case A mass transfer is finished, the primary is losing mass by stellar wind and contracting at the end of core hydrogen burning (dotted line). After this the primary starts with shell hydrogen burning and expands (dash-dotted line).

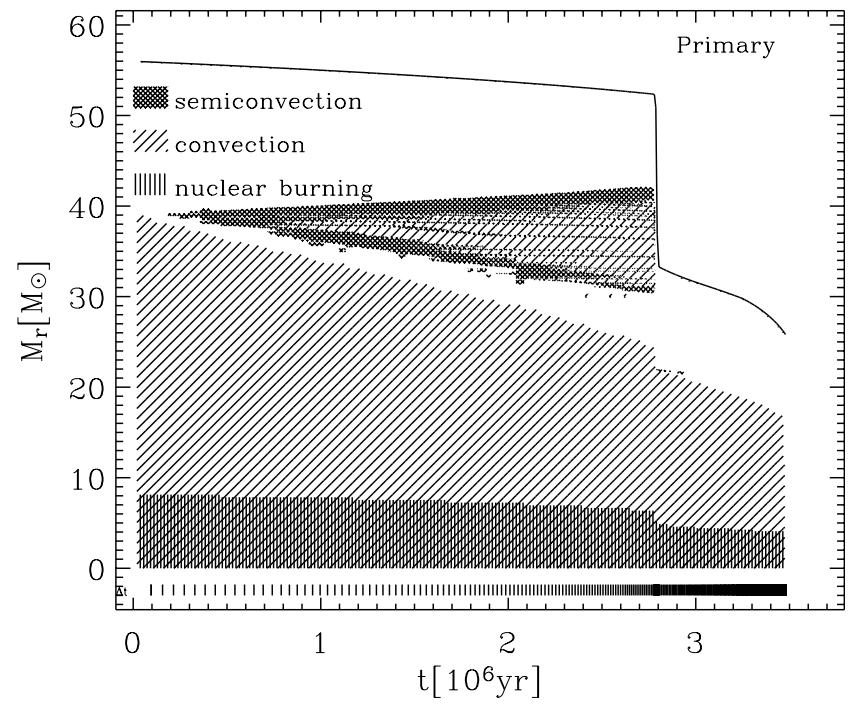

Fig. 4. The evolution of the internal structure of the $56 M_{\odot}$ primary during the core hydrogen burning. Convection is indicated with diagonal hatching and semiconvection with crossed hatching. The hatched area at the bottom indicates nuclear burning. The topmost solid line corresponds to the surface of the star.

During Case AB mass transfer the primary star loses the major part of its hydrogen envelope. After Case AB mass transfer, the primary is a helium core burning star (WR) and the secondary is still a core hydrogen burning $\mathrm{O}$ star. The masses of the modelled WR stars are in the range from $\sim 8 \ldots 18.5 M_{\odot}$ The orbital periods of the modelled $\mathrm{WR}+\mathrm{O}$ systems vary from $\sim 9.5$ to $\sim 20$ days, and the mass ratios are between 0.33 and 0.53 .

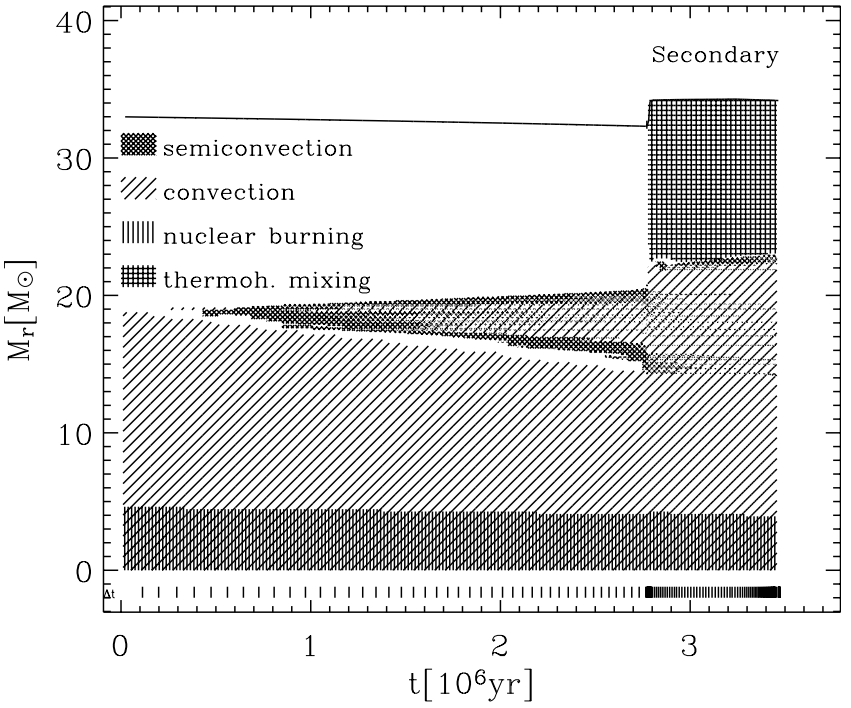

Fig. 5. The evolution of the internal structure of the $33 M_{\odot}$ secondary during core hydrogen burning of the primary. Convection is indicated with diagonal hatching, semiconvection with crossed hatching and thermohaline mixing with straight crossed hatching. The hatched area at the bottom indicates nuclear burning. The topmost solid line corresponds to the surface of the star.

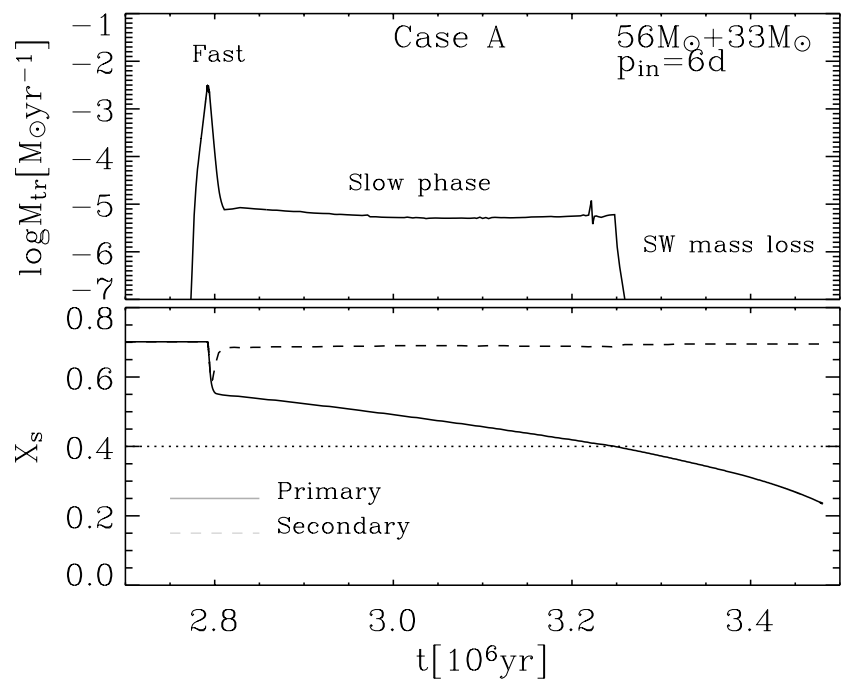

Fig. 6. Upper plot: mass transfer rate during Case A mass transfer in the binary system with $M_{1, \text { in }}=56 M_{\odot}, M_{2, \text { in }}=33 M_{\odot}, p_{\text {in }}=6$ days. Lower plot: the hydrogen surface abundance of the primary (solid line) is decreasing during mass transfer and further due to stellar wind mass loss. The secondary (dashed line) recovered its original surface hydrogen abundance through thermohaline mixing. The primary starts losing mass with WR stellar wind mass loss when its hydrogen surface abundance falls beneath $X_{\mathrm{s}}=0.4$, represented by the dotted line.

\subsection{Relation between initial and WR mass}

The initial mass of helium core of the primary in the binary system depends on a few parameters: initial primary mass, initial period, initial mass ratio and stellar wind mass loss rate. If the primary loses matter due to the mass transfer or stellar wind during core hydrogen burning, it will form a helium core that is less massive than if there was no mass loss. If the initial period is very short, Case A mass transfer will take place very 


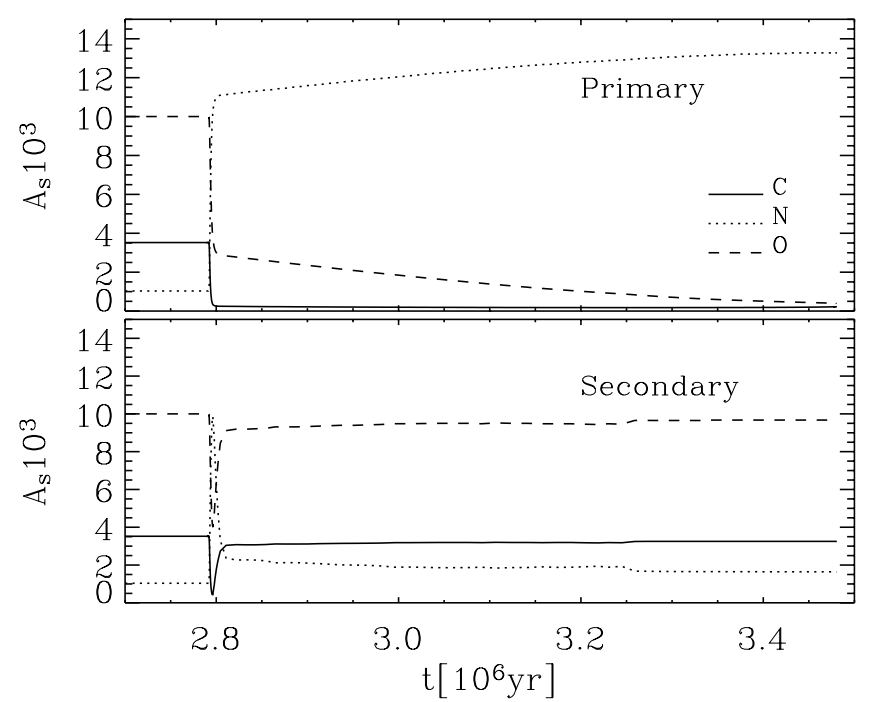

Fig. 7. Surface abundance of carbon (solid line), nitrogen (dotted line) and oxygen (dashed line) of the primary (upper plot) and the secondary (lower plot) in the system with $M_{1, \text { in }}=56 M_{\odot}, M_{2, \text { in }}=33 M_{\odot}$, $p_{\text {in }}=6$ days.

early in the evolution of the primary, so the star will not have time to develop a larger core before it starts losing mass due to Roche lobe overflow. If the initial mass ratio $\left(q=M_{1, \text { in }} / M_{2, \text { in }}\right)$ increases, the mass transfer rate from the primary star increases too and this results in less massive primaries that will evolve into less massive WR stars.

In our models the primary starts losing mass by stellar wind as WR star when its hydrogen surface abundance goes below $X_{\mathrm{s}}=0.4$. However, the observed WR stars in HD 186943, HD 90657 and HD 211853 do not have obvious hydrogen on the surface, so we assume that these WR stars are the result of Case AB mass transfer, with a hydrogen surface abundance of $X_{\mathrm{s}} \approx 0.05$. We also calculated the corresponding WR masses with $X_{\mathrm{s}} \leq 0.01$. We plotted in Fig. 8 the initial WR masses $\left(X_{\mathrm{s}}=0.05\right.$ and $\left.X_{\mathrm{s}} \leq 0.01\right)$ versus the initial primary (progenitor) masses. With "star" symbols we indicated WR stars that originate from binary systems with an initial mass ratio of $q \approx 1.7$ and an initial period $p=3$ days (Table 2: $\mathrm{N} \mathrm{4,} \mathrm{8,} \mathrm{9,}$ 13, 14). Large "star" symbols represent WR stars with $5 \%$ of hydrogen at the surface and small symbols indicate WR stars that have a hydrogen surface abundance of less than $1 \%$.

We derive a relation between the initial primary mass and the initial WR mass (derived as a linear fit) for $p=3$ days and $q \approx 1.7,\left(X_{\mathrm{s}}=0.05\right)$ :

$M_{\mathrm{WR}}=0.24 * M_{1, \text { in }}+0.27$.

We use this relation to estimate the initial parameters of the possible progenitors of the observed $\mathrm{WR}+\mathrm{O}$ binary systems, as already explained in Sect. 3. In the same way, the relation between the initial primary mass and the initial WR mass $\left(X_{\mathrm{s}}<\right.$ 0.01 ) for the same systems is:

$M_{\mathrm{WR}}=0.22 * M_{1, \text { in }}+0.56$.

We also show in Fig. 8 the initial WR masses $\left(X_{\mathrm{S}}=0.05\right.$ for

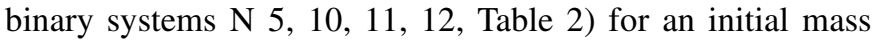

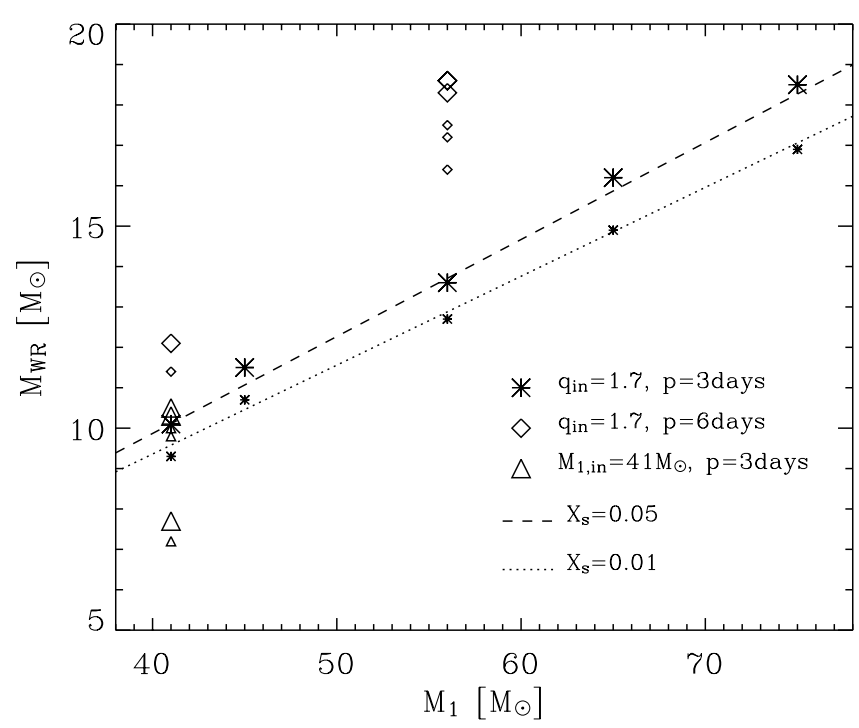

Fig. 8. Initial WR mass as a function of initial (progenitor) mass. Large and small symbols indicate WR stars with hydrogen surface abundance of $X_{\mathrm{s}}=0.05$ and $X_{\mathrm{s}} \leq 0.01$, respectively. Systems with an initial orbital period of $p_{\text {in }}=3$ days and a mass ratio of $q \sim 1.7$ are indicated with star symbols, systems with an initial period of 6 days and $q \sim 1.7$ with diamond symbols, systems with an initial primary $41 M_{\odot}$ and initial period of 3 days with triangle symbols. The dashed line represents a linear fit for systems with an initial period of 3 days and with $X_{\mathrm{s}}=0.05$, and the dotted line represents linear fit for systems with an initial period of 3 days and $X_{\mathrm{s}} \leq 0.01$.

ratio of $\sim 1.7$ and an initial orbital period of 6 days (diamond symbols). We notice that the resulting WR masses are higher than the ones that come out from systems with an initial orbital period of 3 days (see Sect. 5.3). Different "diamond" symbols for the initial primary $56 M_{\odot}$ are for different mass loss rates (see Sect. 5.4). Triangle symbols in Fig. 8 show the initial WR masses for constant initial primary mass, $M_{1, \text { in }}=41 M_{\odot}$, but for different initial mass ratios (see Sect. 5.2).

Note that the WR masses that are the result of early Case A progenitor evolution are significantly lower than ones that are the result of Case B evolution (Wellstein \& Langer 1999), because of the mass transfer from the primary during the core hydrogen burning phase.

\subsection{Influence of the initial mass ratio on the WR mass and orbital period}

During the mass transfer phase, the mass transfer rate increases roughly until the masses of both components are equal. The maximum mass transfer rate during Case $\mathrm{A}$ increases with the increase of the initial mass ratio $\left(M_{1, \text { in }} / M_{2, \text { in }}\right)$ and the resulting WR star is less massive. To analyse the influence of the initial mass ratio on the evolution of the binary system, we compared systems with an initial primary mass of $M_{1 \text {,in }}=41 M_{\odot}$, an initial orbital period of $p_{\text {in }}=3$ days for five different initial mass ratios: 2.05, 2.00, 1.71, 1.52 and 1.37. (Table $2 \mathrm{~N} \mathrm{1,} \mathrm{3,} \mathrm{4,} \mathrm{6,} \mathrm{7,}$ Fig. 9). The system with $q_{\text {in }}=2.05$ enters contact during fast Case A mass transfer. The mass transfer rate in this case is very high $\left(\dot{M} \approx 6 \times 10^{-2} M_{\odot} \mathrm{yr}^{-1}\right)$, the secondary expands, fills its 


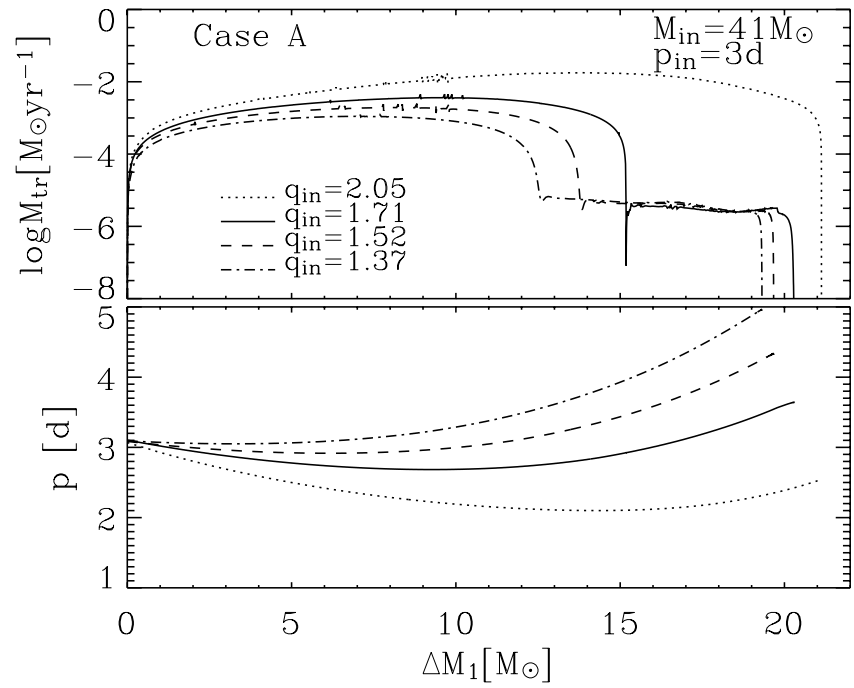

Fig. 9. Mass transfer rate (upper plot) and orbital period (lower plot) during Case A mass transfer as a function of the change of the primary mass for systems with the initial primary $M_{1, \text { in }}=41 M_{\odot}$, initial orbital period $p_{\text {in }}=3$ days and four different initial mass ratios: 2.00 (dotted line), 1.71 (solid line), 1.52 (dashed line) and 1.37 (dash-dotted line). (Table 2: N 3, 4, 6, 7)

Roche lobe and the system enters a contact phase. The system with an initial mass ratio of $q_{\text {in }}=2.00$ loses $\sim 21 M_{\odot}$ during the fast phase of Case A. The maximum mass transfer rate of this system is $\dot{M} \approx 1.8 \times 10^{-2} M_{\odot} \mathrm{yr}^{-1}$. The helium surface abundance of the primary after this mass transfer is $65 \%$, so the primary shrinks, loses mass through a WR stellar wind and there is no slow phase of Case A mass transfer $\left(R_{1}<9 R_{\odot}\right)$. For the other three models $q=1.71,1.52,1.37$, the primaries lose less mass $\left(\sim 15,14,13 M_{\odot}\right.$ respectively) during the fast phase of Case A mass transfer. The helium surface abundances in these systems after fast Case A mass transfer are $~ 30-35 \%$. The primaries expand $\left(R \approx 12-15 R_{\odot}\right)$ on a nuclear time scale and transfer mass to the secondaries (slow phase of Case A).

We can conclude the following: first, if the initial mass ratio is larger, the mass transfer rate from the primary during fast phase Case A mass transfer is higher. Second, if the mass transfer rate is higher, the helium surface abundance of the star increases faster and if it reaches $\approx 58 \%$, the primary starts losing mass with a higher (WR) mass loss rate and slow Case A mass transfer can be avoided.

We also show in Fig. 9 (lower plot) how the period changes during Case A mass transfer for binary systems N 3, 4, 6, 7. Roughly, when the mass is transfered from the more to the less massive star, the binary orbit shrinks, and when the mass is transfered from the less to the more massive star, the orbit widens. If the initial period is close to unity, the absolute difference between stellar masses is small, and more mass is transfered from the less to the more massive star during the evolution of the system. This results in a longer final period after Case A mass transfer. Systems with initial mass ratios of 2.00, $1.71,1.52$ and 1.37 enter Case $A B$ mass transfer with orbital periods of 2.9, 3.9, 4.4 and 5.2 days respectively. However, the final period is also (more significantly) influenced by the

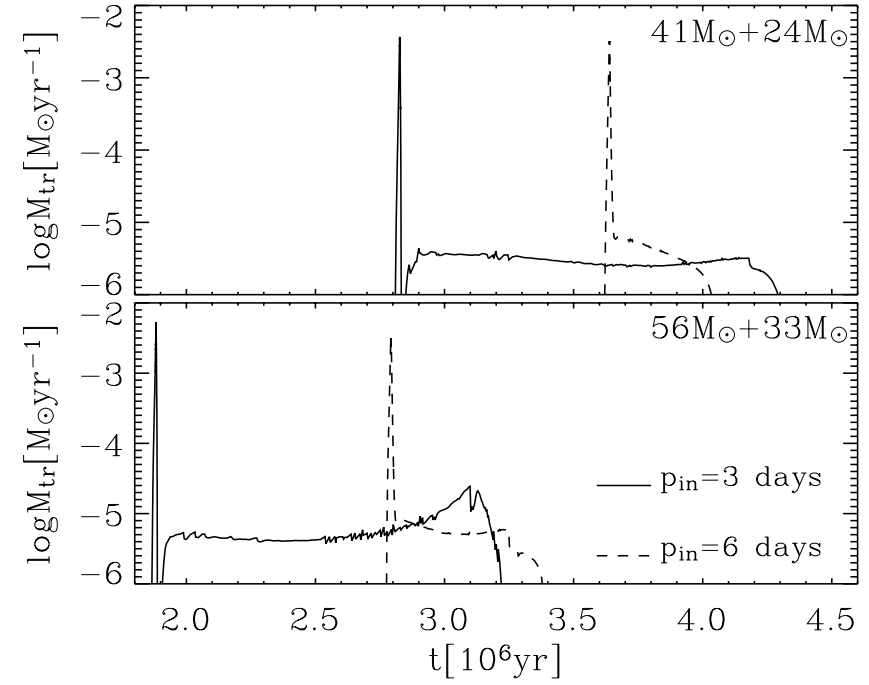

Fig. 10. Mass transfer rate during Case A mass transfer for systems $41 M_{\odot}+24 M_{\odot}$ (upper plot) and $56 M_{\odot}+33 M_{\odot}$ (lower plot) and an initial orbital period of $p_{\text {in }}=3$ days (solid line) and $p_{\text {in }}=6$ days (dashed line). Case A mass transfer starts later in initially wider binary systems, the primary has more time to increase mass of its core and the initial WR star is more massive (Table 2: $\mathrm{N} \mathrm{4,5,9,10).}$

stellar wind mass loss rate and the amount of matter lost from the primary during Case AB mass transfer (see Sect. 5.4).

\subsection{Influence of the initial period on the WR mass}

Depending on the initial orbital period of a binary system, Case A mass transfer phase will start earlier or later in the evolution. If the period is larger, the primary will develop a larger core, before it starts transferring mass onto the secondary, and the resulting WR star will be more massive. To investigate the influence of the initial period, we compare binary systems $41 M_{\odot}+24 M_{\odot}$ and $56 M_{\odot}+33 M_{\odot}$ with $p=3$ days and $p=6$ days (Table 2: $\mathrm{N} 4,5,9,10$ ).

If the initial orbital period increases for 3 days, a $41 M_{\odot}$ star will enter Case A mass transfer $\sim 8 \times 10^{5}$ yr later and a $56 M_{\odot}$ star $\sim 9 \times 10^{5} \mathrm{yr}$ later. So, there are two things to point out: first, the more massive star $\left(56 M_{\odot}\right)$ evolves faster, and second, a 3 days longer initial period postpones Case A mass transfer, for this star, by about $10^{5} \mathrm{yr}$ more than for a $41 M_{\odot}$ star. The net effect is a more significant increase of the convective core (i.e. initial helium core, i.e. initial WR mass), for more massive star, due to the initial orbit widening.

\subsection{Influence of WR mass loss rate on masses and final period}

In our models, we assume that when the star has less than $40 \%$ of hydrogen at the surface, it starts losing mass according to the Hamann et al. (1995) WR mass loss rate, multiplied by factors: $1 / 6,1 / 3$ and $1 / 2$ (Table $2: \mathrm{N} 10,11,12$ ). If the primary is losing more mass by stellar wind during core hydrogen burning, it will develop a less massive helium core. At the same time there will be less matter to be transfered during Roche lobe overflow, so the secondary will accrete less. 


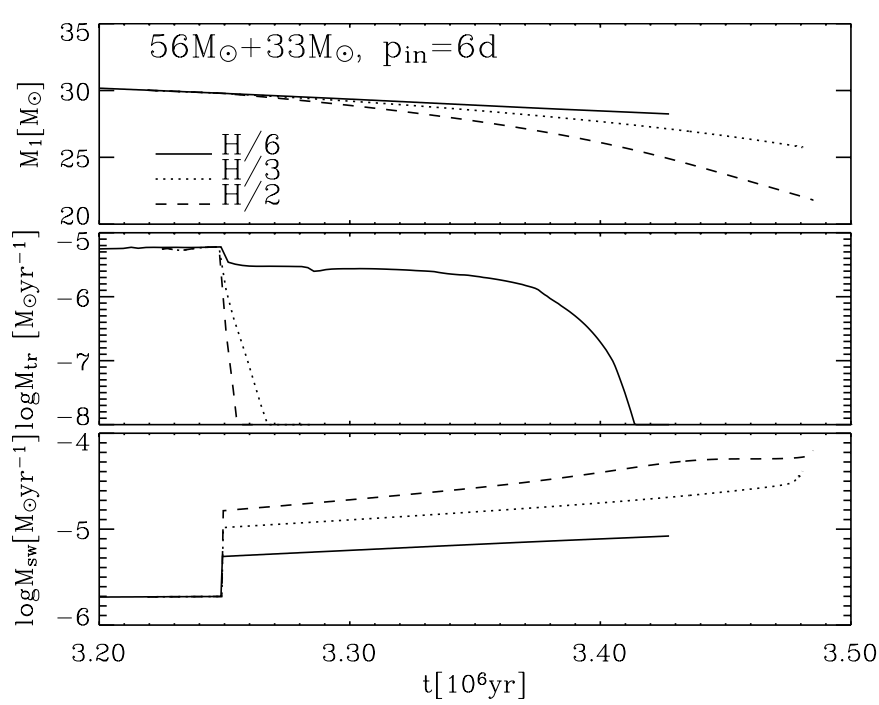

Fig. 11. Primary mass (first plot), mass transfer rate (second plot) and stellar wind mass loss rate from the primary (third plot) until the onset of Case $\mathrm{AB}$ mass transfer for the system $M_{1, \text { in }}=56 M_{\odot}$, $M_{2, \text { in }}=33 M_{\odot}, p_{\text {in }}=6$ days and three different stellar wind mass loss rates (from $\left.X_{\mathrm{s}} \leq 0.4\right)$ : $1 / 6$ (solid line), $1 / 3$ (dotted line) and 1/2 (dashed line) of mass loss rate proposed by Hamann et al. (1995) (Table 2: $\mathrm{N}$ 10, 11, 12).

We show in Fig. 11 the influence of the stellar wind mass loss (Plot c) on the primary mass (Plot a) and mass transfer rate $\left(\right.$ Plot b) of systems with $M_{1, \text { in }}=56 M_{\odot}, M_{2, \text { in }}=33 M_{\odot}$, $p_{\text {in }}=3$ and three different stellar wind mass loss rates (from $\left.X_{\mathrm{s}} \leq 0.4\right): 1 / 6$ (solid line), $1 / 3$ (dotted line) and $1 / 2$ (dashed line) of the mass loss proposed by Hamann et al. (1995). We notice that for higher mass loss rates, the slow phase of Case A stops earlier, due to the decrease of the stellar radius. The orbit is widening due to the stellar wind mass loss and the final period increases with the increasing mass loss rate. However, the orbit is more significantly widening during Case AB mass transfer. The more mass there is to transfer from the primary to the secondary during Case AB mass transfer, the larger the final orbital period. So, if the stellar wind removes most of the hydrogen envelope of the primary, there will be less mass to transfer during Case $\mathrm{AB}$ and the net effect of a higher mass loss rate is a shorter orbital period of the $\mathrm{WR}+\mathrm{O}$ system.

\section{Rotating models}

When mass transfer in a binary system starts, the primary loses matter through the first Lagrangian point $\left(L_{1}\right)$. This matter carries a certain angular momentum that will be transfered to the secondary. If there is an accretion disk, the angular momentum of the transfered matter is assumed to be Keplerian. If there is a direct impact accretion, like in our models, we calculate the angular momentum following a test particle moving through $L_{1}$. This angular momentum spins up the top layers of the secondary star, and angular momentum is transfered further into the star due to rotationally induced mixing processes. Every time the secondary spins up to close to critical rotation it starts losing more mass due to the influence of centrifugal force (Eq. (5)). High mass loss decreases the net accretion efficiency and also removes angular momentum from the secondary star. The secondary star is also spun down by tidal forces that tend to synchronize it with the orbital motion. Wellstein (2001) investigated these processes in binary systems with initial mass ratios close to unity and concluded that the accretion efficiency does not decrease significantly for Case A mass transfer, but in the Case $\mathrm{B}$ the parameter $\beta$ can be significantly decreased by rotation. We present Case A rotating models with larger mass ratio $q=M_{1, \text { in }} / M_{2, \text { in }}=1.7 \ldots 2$ and find that accretion can be significantly decreased during Case A mass transfer. The reason is the following: if the initial mass ratio increases, so does the maximum mass transfer rate $\left(\dot{M}_{\mathrm{mtr}}\right.$ increases roughly until the masses in binary system are equal). If there is more mass transfered from the primary to the secondary, the rotational velocity of the secondary is higher as well as its mass loss, which leads to a smaller accretion efficiency.

We compare the evolution of non-rotating and rotating binary systems on the example $M_{1 \text {,in }}=56 M_{\odot} M_{2 \text {,in }}=33 M_{\odot}$, an initial orbital period of $p=6$ days, and Hamann/3 WR mass loss stellar wind rate (Table 3: $\mathrm{N} \mathrm{6}$ ). The rotating binary system is synchronized as it starts core hydrogen burning and it stays that way until mass transfer starts. The radius of the primary increases during the main sequence phase (from $\sim 10$ to $\sim 25 R_{\odot}$, Fig. 19b), but the rotation of the primary stays synchronized with the orbital period. This is why the rotational velocity of the primary also increases from $\sim 100$ to $\sim 200 \mathrm{~km} \mathrm{~s}^{-1}$ (Fig. 19d). The radius of the rotating primary increases faster than the radius of the non-rotating primary due to the influence of the centrifugal force. The result is that Case A mass transfer starts earlier for the rotating binary system $\left(X_{\mathrm{c}, \text { non }} \approx 80 \%\right)$ then for the corresponding nonrotating one $\left(X_{\mathrm{c}, \text { rot }} \approx 71 \%\right.$, Fig. 15$)$.

When the fast phase of Case A starts, the secondary spins up (Fig. 20d) and stellar wind mass loss rapidly increases $\left(\dot{M}_{\mathrm{sw}} \sim 10^{-3} M_{\odot} \mathrm{yr}^{-1}\right.$, Fig. 20c). The accretion efficiency during this phase in the rotating system is $\beta=0.15$ (Table 3). We see in Fig. 15 that the orbital period after Case A mass transfer of the rotating binary system is shorter than for the non-rotating system (4.5 compared with 6.6 days). The orbital angular momentum of the binary is changing due to mass transfer, mass loss from the system and spin-orbit coupling. The rotating binary system loses more angular momentum and the final orbital period is shorter than in the corresponding non-rotating system. Angular momentum loss in our systems is calculated according to Podsiadlowski et al. (1992) as already mentioned in Sect. 4, and parameter $\alpha$ that determines the efficiency of angular momentum loss is calculated according to Brookshaw \& Tavani (1993). It increases with the mass ratio $M_{2} / M_{1}$ and the ratio between the secondary radius and its Roche radius $R_{2} / R_{12}$. In rotating system the secondary accretes slightly more matter ( $\bar{\beta}=0.15$ ) compared to $\beta=0.1$ in non-rotating systems, so the mass ratio $M_{2} / M_{1}$ is larger in the rotating system. Second, the secondary is spinning fast and its radius is larger than in the non-rotating case, and so is the ratio $R_{2} / R_{12}$. The result is that the angular momentum is more efficiently removed from the system in the rotating binary system. After the fast phase of Case A mass transfer, the two primaries, non-rotating and rotating, have almost the same mass $\sim 34 M_{\odot}$ and helium surface abundance $Y_{\mathrm{s}} \sim 44 \%$. However, since the orbital periods are 


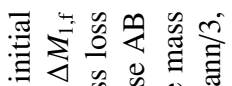

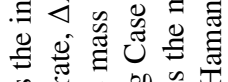

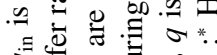

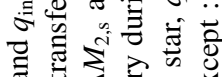

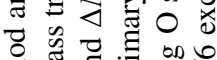
흘

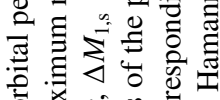

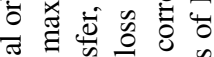

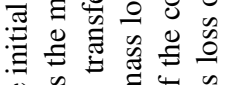
o.

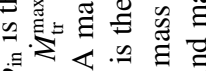

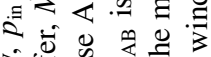

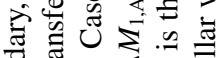

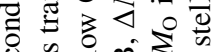
皜

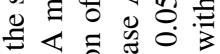

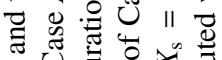
능

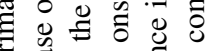

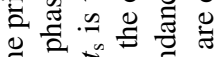
을 흔

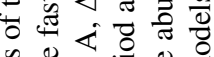

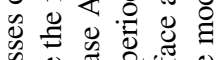

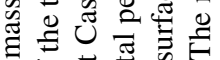

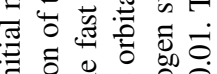

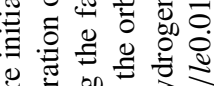

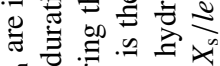

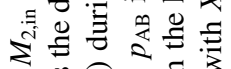

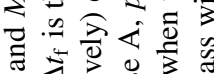

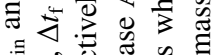
s)

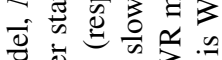
을

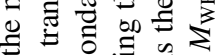

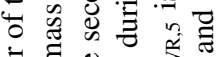

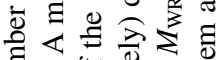

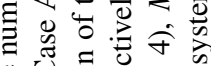

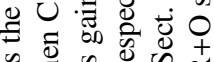

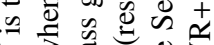

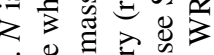

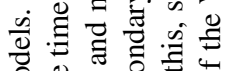

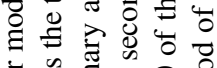

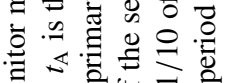

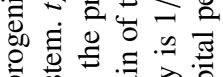

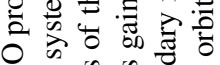

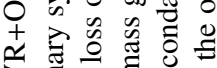

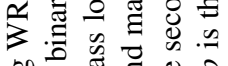
of

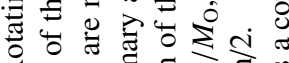

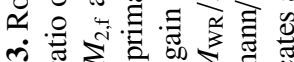

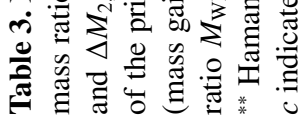

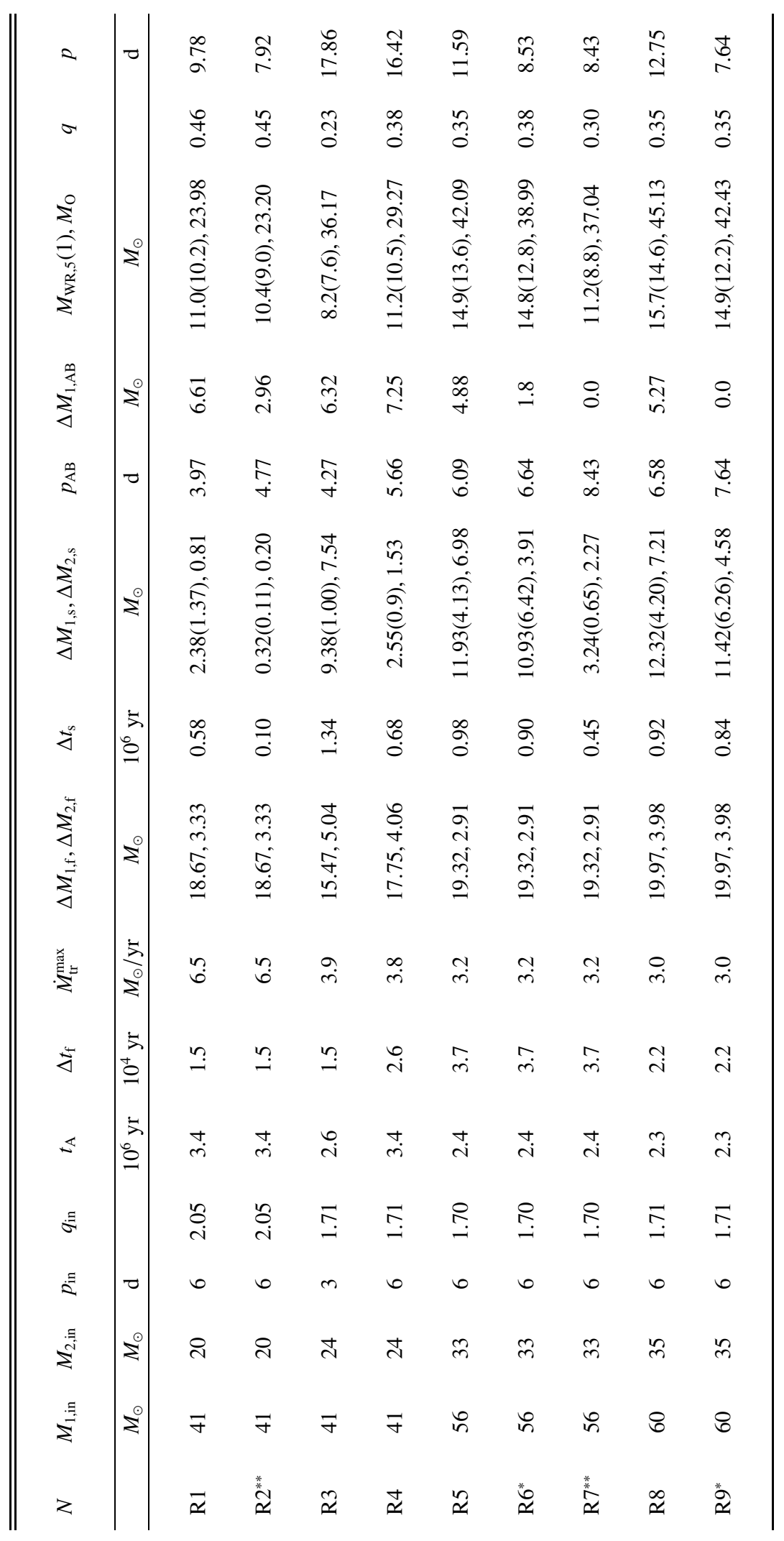


Table 4. Comparison of resulting WR masses and orbital periods from non-rotating and rotating binary systems with the same initial parameters. $M_{1, \text { in }}, M_{2, \text { in }}$ are initial primary and secondary mass, $p_{\text {in }}$ is the initial orbital period, $M_{\mathrm{WR}, 5}, M_{\mathrm{WR}, 1}$ are WR masses at $X_{\mathrm{s}}=0.05$ and $X_{\mathrm{s}} \leq 0.01$ respectively and $p$ is the orbital period in the initial $\mathrm{WR}+\mathrm{O}$ system where the hydrogen surface abundance of WR star is $X_{\mathrm{s}}=0.05$. Systems are modelled with WR stellar wind mass loss $\mathrm{H} / 6$ except ${ }^{*}$ which are done with $\mathrm{H} / 3,{ }^{\mathrm{R}}$ indicates rotating models.

\begin{tabular}{lcccc}
\hline \hline $\begin{array}{l}M_{1, \text { in }}+M_{2, \text { in }} \\
{\left[M_{\odot}\right]}\end{array}$ & $\begin{array}{c}p_{\text {in }} \\
{[\mathrm{d}]}\end{array}$ & $\begin{array}{c}M_{\mathrm{WR}, 5} \\
{\left[M_{\odot}\right]}\end{array}$ & $\begin{array}{c}M_{\mathrm{WR}, 1} \\
{\left[M_{\odot}\right]}\end{array}$ & $\begin{array}{c}p \\
{[\mathrm{~d}]}\end{array}$ \\
\hline $41+20$ & 6 & 11.8 & 11.2 & 12.6 \\
$41+20^{\mathrm{R}}$ & 6 & 11.0 & 10.2 & 9.8 \\
$41+24$ & 6 & 12.1 & 11.4 & 21.5 \\
$41+24^{\mathrm{R}}$ & 6 & 11.2 & 10.5 & 16.4 \\
$56+33^{*}$ & 6 & 18.6 & 17.5 & 13.8 \\
$56+33^{* \mathrm{R}}$ & 6 & 14.9 & 13.6 & 8.5 \\
\hline
\end{tabular}

different, so are the radii of the primaries $\left(\sim 18 R_{\odot}\right.$ for the rotating and $\sim 23 R_{\odot}$ for the non-rotating case).

When the fast phase of Case $\mathrm{A}$ is finished, the non-rotating primary has still $\sim 20 \%$ of hydrogen to burn $\left(\sim 7 \times 10^{5} \mathrm{yr}\right)$, and the rotating primary has $\sim 10 \%$ more than that $\left(\sim 1.2 \times 10^{6} \mathrm{yr}\right)$. When the surface hydrogen abundance is less than $40 \%$, the primaries start losing mass as WR stars, i.e., their stellar wind mass loss rate increases. Since the rotating primary has more time to spend on the main sequence, it also has more time to lose mass by WR stellar wind mass loss $\left(7.2 \times 10^{5} \mathrm{yr}\right.$ compared with $2.5 \times 10^{5} \mathrm{yr}$ for non-rotating system).The result is that the non-rotating primary enters Case $\mathrm{AB}$ mass transfer as a $\sim 26 M_{\odot}$ star with $Y_{\mathrm{s}}=0.75$, while the rotating one is a $\sim 17 M_{\odot}$ star with $Y_{\mathrm{s}}=0.90$. Clearly, the rotating primary has less hydrogen in its envelope, i.e. less mass to transfer to the secondary during Case $\mathrm{AB}$ mass transfer, and the orbit widens less than in the non-rotating system. We can draw the conclusion that if rotation is included in our calculations, the initial WR mass is smaller and the orbital period of the $\mathrm{WR}+\mathrm{O}$ system is shorter than in the corresponding non-rotating system (Table 4).

We present in Fig. 12 the evolutionary tracks of the rotating primary and secondary in the HR diagram. Both stars are core hydrogen burning stars (dashed line, Fig. 12), but since the primary is more massive, it evolves faster and fills its Roche lobe, so the system enters Case A mass transfer (solid line, Fig. 12). The primary loses matter quickly with a high mass transfer rate $\left(\dot{M}_{\mathrm{tr}}^{\max } \approx 3.2 \times 10^{-3} M_{\odot} \mathrm{yr}^{-1}\right)$ and its luminosity decreases (Fig. 12). At the same time the secondary accretes matter and its luminosity increases, but due to change in rotational velocity (Fig. 20d) its radius and effective temperature are changing as well (Figs. 12d, 20a,b). During fast Case A mass transfer the primary lost $\sim 19 M_{\odot}$ and the secondary accreted $15 \%$ of that matter. After the fast mass transfer, the primary is still burning hydrogen in its core and is still expanding, so slow Case A mass transfer takes place. After the primary starts losing mass with a WR stellar wind mass loss rate $\left(X_{\mathrm{s}}<0.4\right)$ its radius will decrease and the slow phase of Case A stops (Fig. 19c). However, the primary continues expanding on the nuclear time scale (Fig. 19b) and it fills its Roche lobe once again (Fig. 15, upper plot). At the end of

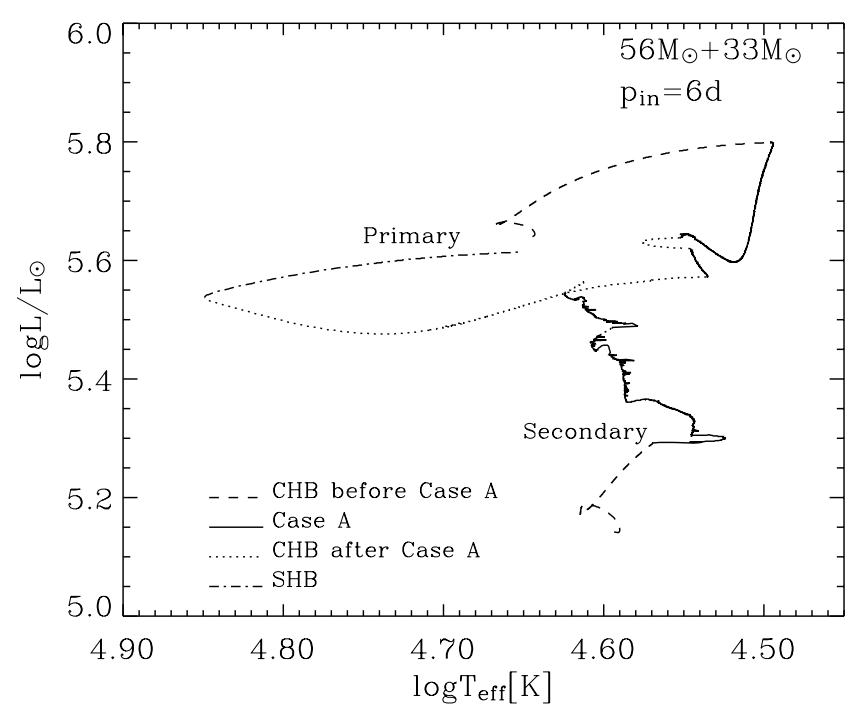

Fig. 12. HR diagram of the initial system $M_{1, \text { in }}=56 M_{\odot}$, $M_{2, \text { in }}=33 M_{\odot}, p_{\text {in }}=6$ days with rotation. Both stars are core hydrogen burning (dashed line) until Case A mass transfer starts (solid line). The primary is losing mass and its luminosity decreases. At the same time the secondary is accreting matter and expanding, becoming more luminous. After Case A mass transfer is finished, the primary is losing mass by stellar wind and contracting at the end of core hydrogen burning (dotted line). After this the primary starts shell hydrogen burning and expands (dash-dotted line).

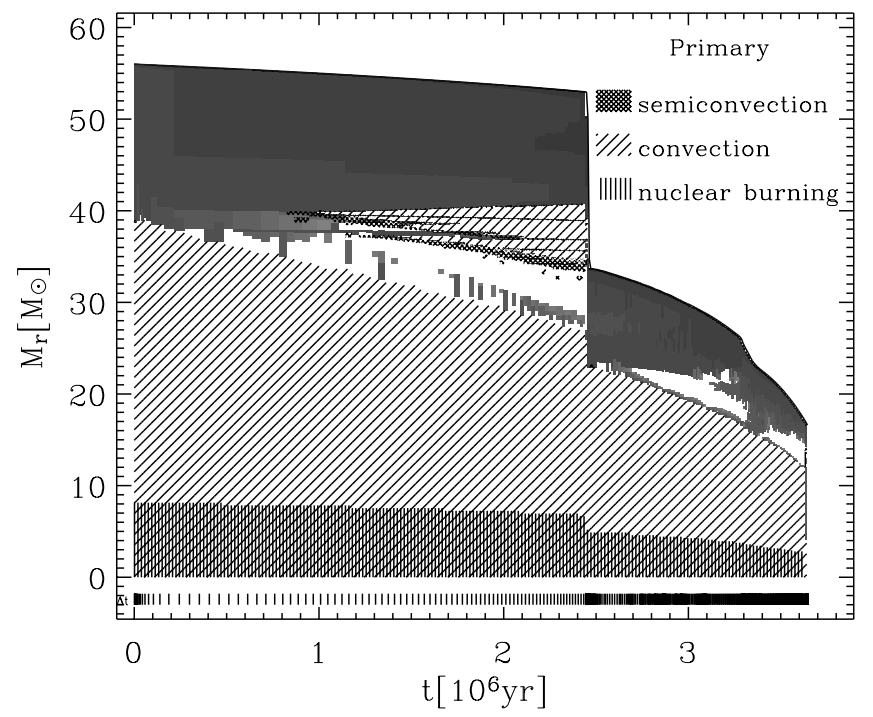

Fig. 13. The evolution of the internal structure of the rotating $56 M_{\odot}$ primary during core hydrogen burning. Convection is indicated with diagonal hatching and semiconvection with crossed hatching. The hatched area at the bottom indicates nuclear burning. Gray shaded areas represent regions with rotationally induced mixing (intensity is indicated with different shades, the darker the colour, the stronger rotational mixing). The topmost solid line corresponds to the surface of the star.

core hydrogen burning the primary contracts (effective temperature increases) and thus RLOF stops. This phase is presented in Fig. 12 with a dotted line. When hydrogen starts burning in a shell, the primary star expands (dash-dotted line, Fig. 12), fills its Roche lobe and Case AB mass transfer starts. 


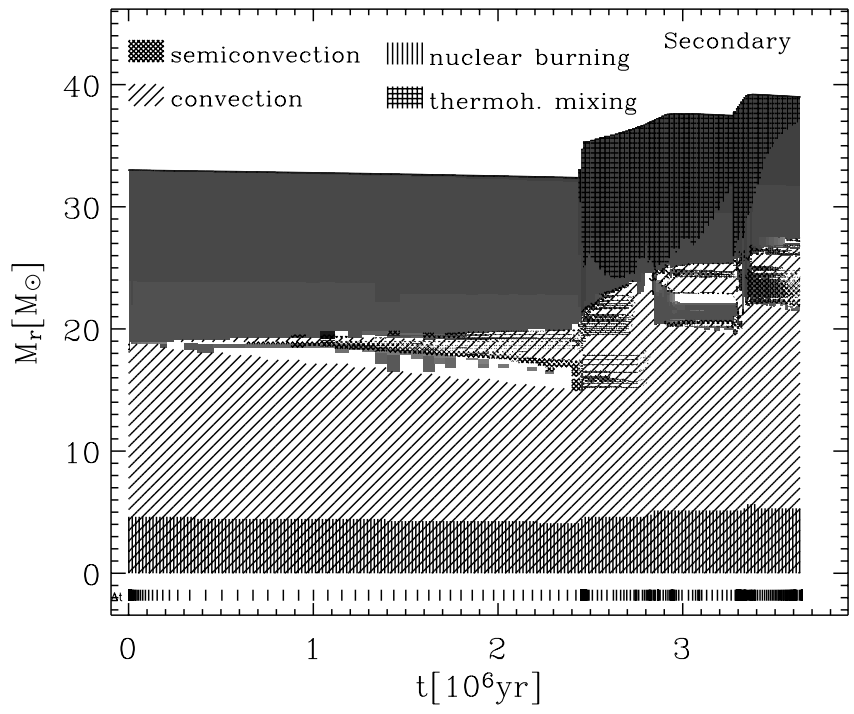

Fig. 14. The evolution of the internal structure of the rotating $33 M_{\odot}$ secondary during core hydrogen burning of the primary. Convection is indicated with diagonal hatching, semiconvection with crossed hatching and thermohaline mixing with straight crossed hatching. The hatched area at the bottom indicates nuclear burning. Gray shaded areas represent regions with rotationally induced mixing (intensity is indicated with different shades, the darker the colour, the stronger rotational mixing). The topmost solid line corresponds to the surface of the star.

The initial helium core masses are $18.6 M_{\odot}$ for the nonrotating and $14.8 M_{\odot}$ for the rotating primary. When Case AB mass transfer starts, the orbital periods are $7.9 \mathrm{~d}$ and $6.6 \mathrm{~d}$ for the non-rotating and the rotating system respectively (Fig. 15, lower plot). The non-rotating primary loses $\sim 7 M_{\odot}$ and the rotating one $\sim 2 M_{\odot}$ during Case $\mathrm{AB}$. When there is more mass to be transfered from the less to the more massive star in a binary system, the orbit widens more and the final orbital period is longer.

Figures 13 and 14 show the structure of the primary and the secondary before Case AB mass transfer. The primary loses large amounts of matter during the fast phase of Case A mass transfer $\left(\sim 20 M_{\odot}\right)$, and its convective core becomes less than half of its original mass. At the same time, the secondary accretes matter from the primary and the heavier elements are being relocated by thermohaline mixing. Figures 16 and 17 show surface abundances of the primary and the secondary. The secondary is accreting material from the primary and its surface abundances change due to this, but also due to thermohaline and rotational mixing.

Figure 18 shows the orbital angular momentum of the system and the spin periods of both components. The orbital angular momentum of the system decreases rapidly due to mass loss from the system during fast Case A mass transfer, and then further due to stellar wind mass loss. The primary slows down rapidly during fast Case $\mathrm{A}$ and further due to stellar wind mass loss. The secondary spins up due to the accretion from the primary during fast Case A mass transfer and then slows down due to stellar wind mass loss. It spins up again during slow Case A mass transfer.

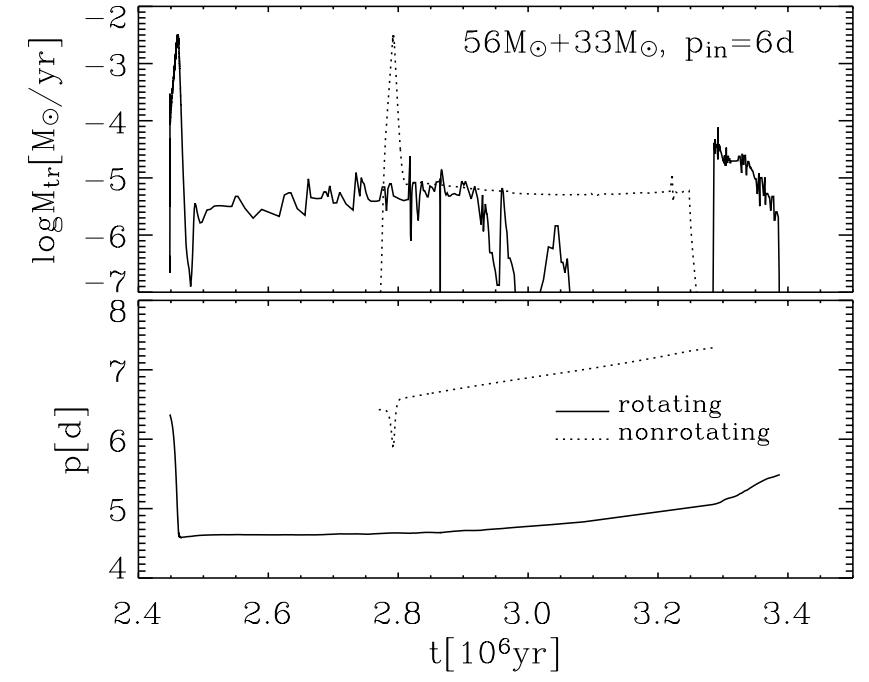

Fig. 15. Upper plot: the mass transfer rate during Case A mass transfer in the binary systems with $M_{1, \text { in }}=56 M_{\odot}, M_{2, \text { in }}=33 M_{\odot}, p_{\text {in }}=6$ days with (solid line) and without rotation (dotted line). Lower plot: orbital period evolution in rotating and non-rotating system.

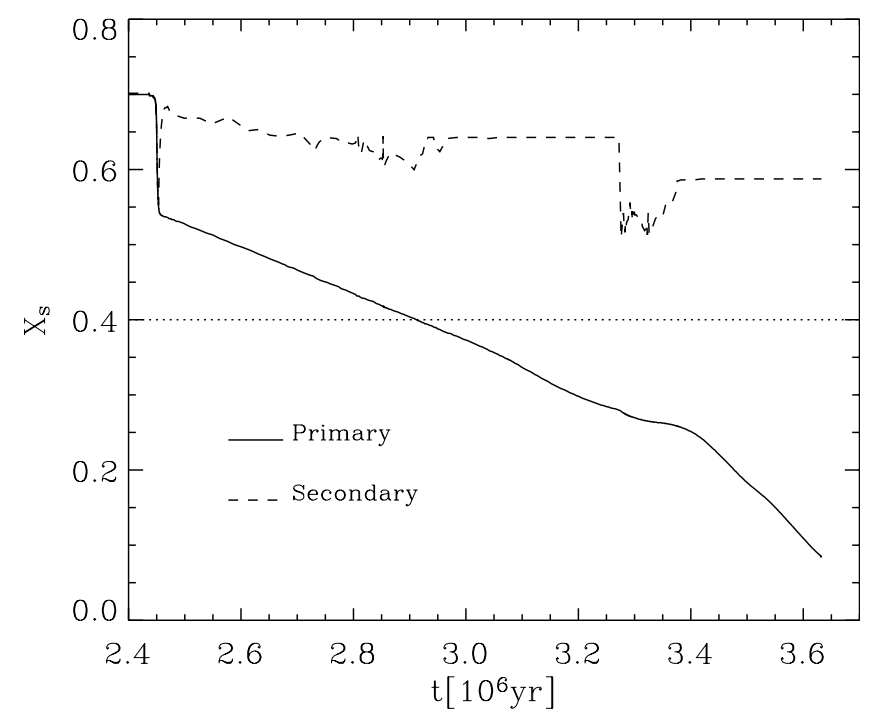

Fig. 16. The hydrogen surface abundance (solid line) in the primary in system with $M_{1, \text { in }}=56 M_{\odot}, M_{2, \text { in }}=33 M_{\odot}, p_{\text {in }}=6$ days is decreasing during mass transfer and further due to stellar wind mass loss. The secondary (dashed line) decreases its hydrogen surface abundance due to mass transfer. The dotted line indicates a hydrogen abundance of 0.4 , where the primary starts losing mass with a WR stellar wind.

The masses of modelled WR stars are in the range from $\sim 11 M_{\odot}$ to $\sim 15.7 M_{\odot}$. Period of modelled $\mathrm{WR}+\mathrm{O}$ systems vary from $\sim 7.6$ to $\sim 12.7$ days and mass ratios are between 0.35 and 0.46 (Table 3 ).

\subsection{Influence of rotation on the accretion efficiency}

We show in Table 5 average accretion efficiencies of rotating binary systems during different mass transfer phases, and total average values with and without stellar wind mass loss from the primary included. During fast Case A mass transfer, the primary stars are losing matter with very high mass transfer 


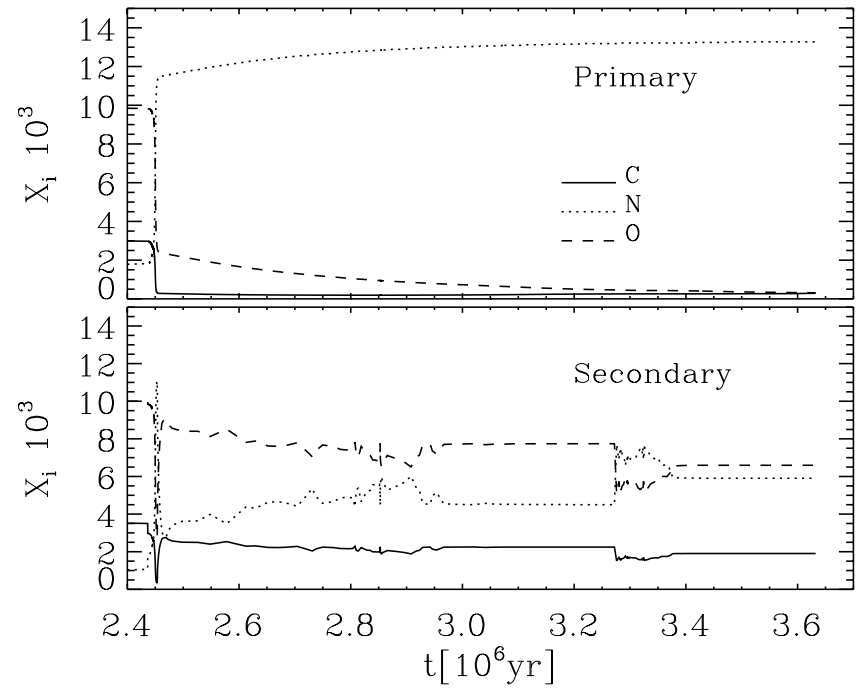

Fig. 17. Surface abundance of carbon (solid line), nitrogen (dotted line) and oxygen (dashed line) in the primary (upper plot) and the secondary (lower plot), in the system with $M_{1, \text { in }}=56 M_{\odot}, M_{2, \text { in }}=$ $33 M_{\odot}, p_{\text {in }}=6$ days. The secondary abundances are changed due to mass transfer of matter from the primary, thermohaline mixing and rotational mixing.

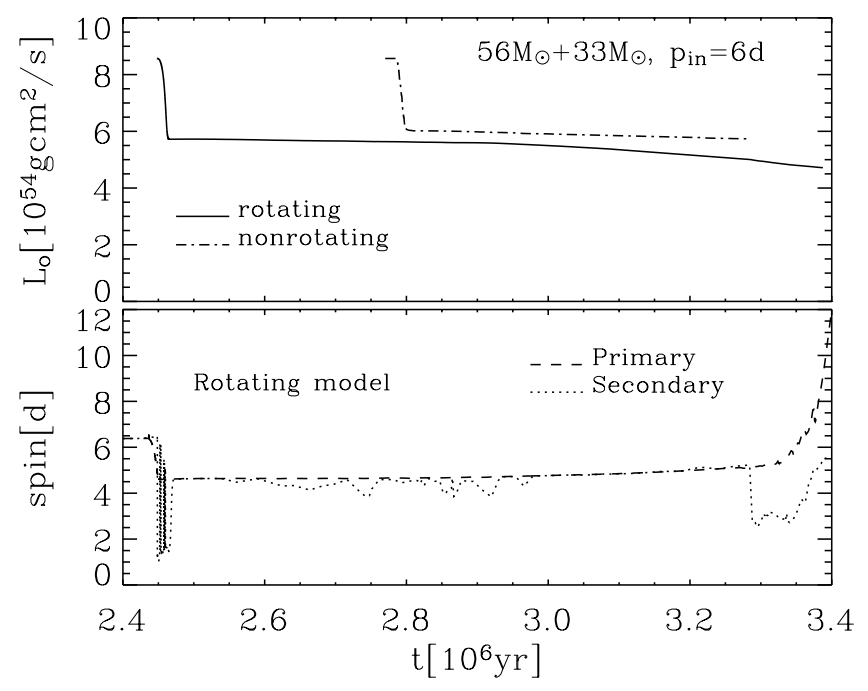

Fig. 18. The orbital angular momentum (upper plot) of the nonrotating (dotted line) and the rotating (solid line) binary systems with $M_{1, \text { in }}=56 M_{\odot}, M_{2, \text { in }}=33 M_{\odot}, p_{\text {in }}=6$ days, decreases rapidly due to mass loss from the system during fast Case A mass transfer and then further due to stellar wind mass loss. Spin period (lower plot) of the primary (dashed line) and the secondary (dash-dotted line) in the above mentioned rotating binary system.

rates $\left(3 \ldots 6.5 \times 10^{-3} M_{\odot} \mathrm{yr}^{-1}\right)$. The angular momentum of surface layers in the secondary increases fast, they spin up to close to the critical rotation and start losing mass with high mass loss rate $\left(\sim 10^{-3} M_{\odot}\right)$. The average accretion efficiency during fast Case A in our models is $15-20 \%$. Since this phase takes place on the thermal time scale, stellar wind mass loss from the primary is negligible during this phase.

Slow Case A mass transfer takes place on the nuclear time scale. The primary stars start losing their mass due to a WR stellar wind when their surfaces become hydrogen deficient

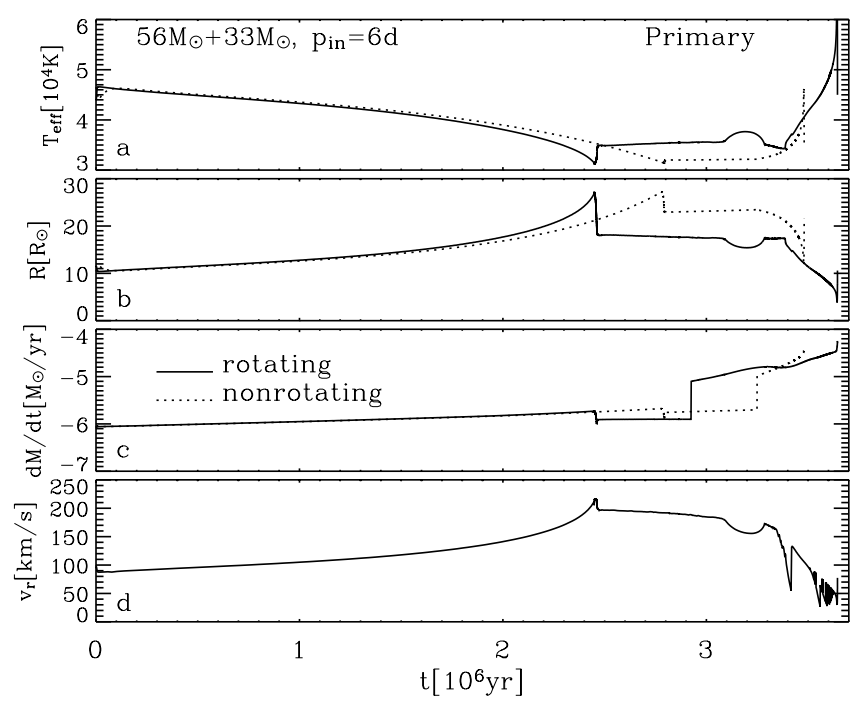

Fig. 19. Effective temperature (plot a)), stellar radius (plot b)), stellar wind mass loss rate (plot c)) and rotational velocity (plot d)) of the primary star in the non-rotating (dotted line) and rotating (solid line) binary system with $M_{1, \text { in }}=56 M_{\odot}, M_{2, \text { in }}=33 M_{\odot}, p_{\text {in }}=6$ days.

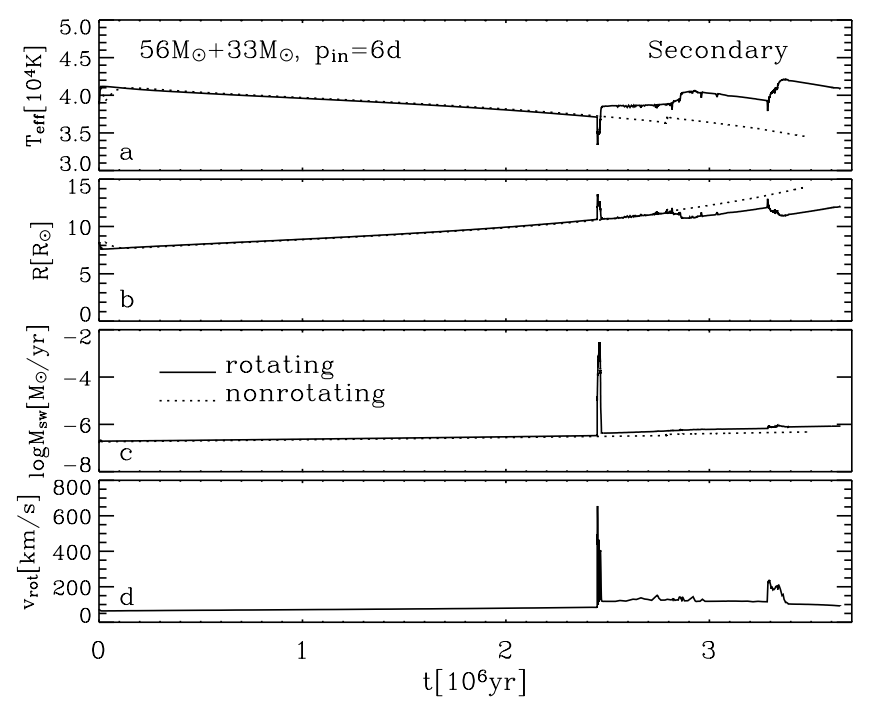

Fig. 20. Effective temperature (plot a)), stellar radius (plot b)), stellar wind mass loss rate (plot c)) and rotational velocity (plot d)) of the secondary star in the non-rotating (dotted line) and rotating (solid line) binary system with $M_{1, \text { in }}=56 M_{\odot}, M_{2, \text { in }}=33 M_{\odot}, p_{\text {in }}=6$ days.

$\left(X_{\mathrm{s}}<0.4\right)$. The WR stellar wind mass loss rates are of the order of: $\dot{M} \sim 10^{-5} M_{\odot} \mathrm{yr}^{-1}$, and we have to take into account stellar wind mass loss of the primary during slow Case A. We calculate the mass loss of the primary only due to mass transfer and total mass loss including stellar wind mass loss, and the two corresponding average accretion efficiencies. If we calculate $\bar{\beta}_{\mathrm{s}}$ only for mass transfer, we notice that the slow Case $\mathrm{A}$ is almost a conservative process. The average mass transfer rates are $\sim 10^{-6} M_{\odot} \mathrm{yr}^{-1}$ and the secondary stars are able to accrete almost everything without spinning up to critical rotation.

Figure 21 shows how mass transfer rate, accretion rate and $\beta$ change in the rotating model with $56 M_{\odot}+33 M_{\odot}$, $p_{\text {in }}=6$ days (WR mass loss Hamann/3) depending on the amount of matter lost by the primary. We also see in this 


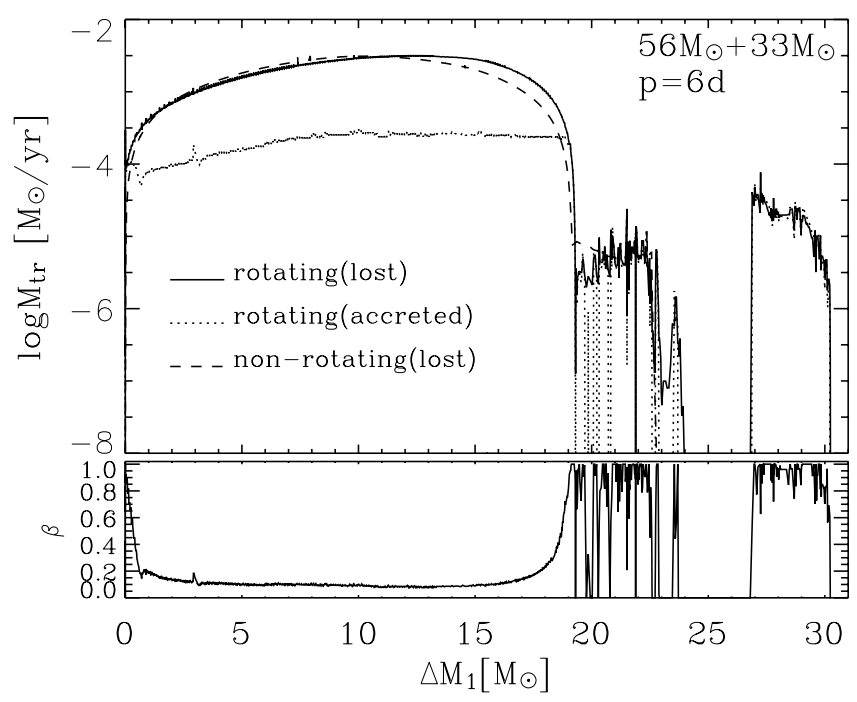

Fig. 21. Upper plot: mass transfer (solid line) and accretion rate (dotted line) of the rotating initial system $56 M_{\odot}+33 M_{\odot}, p=6$ days. Dashed line represent mass transfer rate in the corresponding nonrotating binary. Lower plot: accretion efficiency of the secondary taking into account matter lost by the primary only due to mass transfer.

figure the mass transfer rate from the primary in the nonrotating case. We can notice in the upper plot, what we previously discussed, that during most of the fast Case A mass transfer, the mass accretion rate of the secondary is about one order of magnitude lower than the mass loss rate of the primary. The primary loses $\sim 19.3 M_{\odot}$ during the fast phase and the secondary gains $\sim 2.9 M_{\odot}$, which means that on average $\sim 15 \%$ of the mass has been accreted. However, the mass loss of the primary due to mass transfer during the slow phase is $\sim 4.5 M_{\odot}$, and the secondary accretes $\sim 3.9 M_{\odot}$ which means that $\bar{\beta}_{\mathrm{s}} \sim 0.87$. If we take into account stellar wind mass loss of the primary stars, the average accretion efficiencies are lower. For example, the total mass loss of the primary during slow Case A mass transfer, including the stellar wind, in the previous example is $\sim 10.9 M_{\odot}$, which means that $\bar{\beta}_{\mathrm{s}}^{\mathrm{w}} \approx 0.36$. We neglected accretion during Case AB mass transfer since Wellstein (2001) showed that it is inefficient, and since the primary stars in the modelled systems have relatively low mass hydrogen envelopes, and masses of secondary stars will not significantly change due to this mass transfer. (However, let us not forget that even the accretion of very small amounts of matter can be important for spinning up the secondary's surface layers and making it rotate faster than synchronously in a WR $+\mathrm{O}$ binary system.) Also, since this mass transfer takes place on the thermal time scale, stellar wind mass loss can be neglected.

Finally, we can estimate the total mass loss from the binary systems including stellar wind, or only due to mass transfer, and calculate corresponding values of $\beta$. In the binary systems we modelled, the primary stars lose between $30 M_{\odot}$ and $45 M_{\odot}$ due to mass transfer and stellar wind, until they ignite helium in their core. The amount of lost mass increases with initial mass. At the same time the secondaries accrete $3 \ldots 10 M_{\odot}$. This means that in most cases $80 \ldots 90 \%$ of the mass lost by the primary leaves the binary system. On the other hand, the primary stars lose $\sim 20 \ldots 30 M_{\odot}$ only due to mass transfer, so the
Table 5. Mass loss from binary systems. $N$ is number of the model corresponding to Table 3. $\bar{\beta}_{\text {fast }}$ is the average accretion efficiency of the secondary during the fast phase of Case A mass transfer. $\bar{\beta}_{\text {slow }}$ is the accretion efficiency of the secondary during the slow phase of Case $\mathrm{A}$ mass transfer taking into account matter lost by the primary only due to the mass transfer. $\bar{\beta}_{\text {slow }}^{\text {wind }}$ is the average accretion efficiency of the secondary during the slow phase of Case A mass transfer taking into account matter lost by the primary due to the mass transfer and stellar wind. $\bar{\beta}$ is the average accretion efficiency of the secondary during the progenitor evolution of $\mathrm{WR}+\mathrm{O}$ binary system taking into account matter lost by the primary only due to the mass transfer and $\bar{\beta}_{\mathrm{mtr}}^{\text {wind }}$ taking also into account stellar wind mass loss of the primary.

\begin{tabular}{lccccc}
\hline \hline$N$ & $\bar{\beta}_{\text {fast }}$ & $\bar{\beta}_{\text {slow }}$ & $\bar{\beta}_{\text {slow }}^{\text {wind }}$ & $\bar{\beta}$ & $\bar{\beta}^{\text {wind }}$ \\
\hline $\mathrm{R} 1$ & 0.18 & 0.80 & 0.35 & 0.15 & 0.13 \\
$\mathrm{R} 2$ & 0.18 & 0.95 & 0.65 & 0.15 & 0.10 \\
$\mathrm{R} 3$ & 0.33 & 0.90 & 0.80 & 0.40 & 0.37 \\
$\mathrm{R} 4$ & 0.23 & 0.94 & 0.60 & 0.20 & 0.18 \\
$\mathrm{R} 5$ & 0.15 & 0.90 & 0.58 & 0.28 & 0.22 \\
$\mathrm{R} 6$ & 0.15 & 0.87 & 0.36 & 0.23 & 0.14 \\
$\mathrm{R} 7$ & 0.15 & 0.88 & 0.70 & 0.18 & 0.09 \\
$\mathrm{R} 8$ & 0.20 & 0.88 & 0.58 & 0.30 & 0.23 \\
$\mathrm{R} 9$ & 0.20 & 0.89 & 0.40 & 0.30 & 0.16 \\
\hline
\end{tabular}

average accretion of secondary stars in our models is between 15 and $30 \%$.

\section{Comparison with observations}

Our rotating models give generally similar results as our nonrotating models for $\beta=0.1$.

The rotating binary systems $\mathrm{R} 6\left(56 M_{\odot}+33 M_{\odot}, p=6\right.$ days, Hamann/3 WR mass loss) and R1 and R2 (41 $M_{\odot}+$ $20 M_{\odot}, p=6$ days) agree quite well with the observed systems HD 186943 and HD 90657, as well as the non-rotating systems $\mathrm{N} 11$ and $\mathrm{N} 12\left(56 M_{\odot}+33 M_{\odot}, p=6\right.$ days; WR mass loss rate Hamann/2 and Hamann/3). The system R6 evolves into a $\mathrm{WR}+\mathrm{O}$ configuration with $15 M_{\odot}+39 M_{\odot}$ and $p=8.5$ days. I.e., its masses and period are close to those found in HD 186943 and HD 90657, even though its mass ratio of 0.38 is somewhat smaller than what is observed. Systems R1 and $\mathrm{R} 2$ evolve into a $11 M_{\odot}+24 M_{\odot} \mathrm{WR}+\mathrm{O}$ system with a 9.8 day orbital period. I.e., period and mass ratio (0.46) agree well with the observed systems, but the stellar masses are somewhat smaller than observed (cf. Sect. 2). Systems N11 and $\mathrm{N} 12$ evolve into a WR+O system of $19 M_{\odot}+35 M_{\odot}$ with an orbital period of $12 \ldots 14$ days. In this case, both masses and the mass ratio (0.53) agree well with the observed ones, but the orbital period is slightly too large. I.e., although none of our models is a perfect match of HD 186943 or HD 90657 - which to find would require many more models, however, might not teach us very much - it is clear form these results that both systems can in fact be well explained through highly inefficient Case A mass transfer.

The situation is more difficult with HD 211853 (GP Cep): neither the models with nor those without rotation reproduce it satisfactory. HD 211853 has the shortest period (6.7 d) 
and largest mass ratio $(0.54)$ of the three chosen Galactic $\mathrm{WR}+\mathrm{O}$ binaries. While we can not exclude that a Case A model of the kind presented here can reproduce this systems, especially the small period makes it appear more likely that this system has gone through a contact phase: contact would reduce the orbital angular momentum, and increase the mass loss from the system, i.e. result in a larger WR/O mass ratio (Wellstein et al. 2001). This reasoning is strengthened by the consideration that, in contrast to HD 186943 or HD 90657, the WR star in HD 211853 is of spectral type WN6/WNC. I.e., as this spectroscopic signature is not interpreted in terms of a binary nature of the WR component, but rather by assuming that the WR star is in the transition phase from the WN to the WC stage (Massey \& Grove 1989; Langer 1991). This implies that the WR star in HD 211853 must have already lost several solar masses of helium-rich matter, which causes the orbit to widen. For example, system R6, which evolved into a $14.8 M_{\odot}+39.0 M_{\odot} \mathrm{WR}+\mathrm{O}$ system with $p=8.53$ days, evolves into a $\mathrm{WC}+\mathrm{O}$ system after losing $\sim 5 M_{\odot}$ more from the Wolf-Rayet star, which increases its orbital period by $\sim 3$ days. I.e., HD 211853 might have entered the $\mathrm{WR}+\mathrm{O}$ stage with an orbital period of about 4 days, which would put it together with the shortest period WR binaries like CX Cep or CQ Cep whose periods are 2.1 and 1.64 days respectively.

During the evolution of $\mathrm{WR}+\mathrm{O}$ binary system, the primary loses mass due to WR stellar wind mass loss. WR stellar wind mass loss of the primary decreases mass ratio of the system and increases the orbital period, which means that, for example, WC + O binary system HD $63099\left(M_{\mathrm{WR}}=9 M_{\odot}, M_{\mathrm{O}}=32 M_{\odot}\right.$ and $p=14$ days) could have evolved into present state through a WN+O binary system with $q=0.5$.

\section{Conclusions}

In an effort to constrain the progenitor evolution of the three WN+O binaries HD 186943, HD 90657, and HD 211853, we calculated the evolution of non-conservative Case A binary systems with primaries $M_{1, \mathrm{i}}=41 \ldots 65 M_{\odot}$ and initial mass ratios between 1.7 and 2 until the $\mathrm{WN}+\mathrm{O}$ stage. We performed binary evolution calculations neglecting rotational processes in the two stellar components, and assuming a constant mass accretion efficiency of $10 \%$ for all three phases of the mass transfer, fast Case A, slow Case A, and Case AB. Those models could match two of the three systems reasonably well, while HD 211853, which has the shortest orbital period, the largest mass ratio, and a WN/WC Wolf-Rayet component, was found to be not well explained by contact-free evolutionary models: While models with shorter initial orbital periods result in short periods during the $\mathrm{WR}+\mathrm{O}$ stage, the initial WR mass is decreasing at the same time, which leads to smaller initial $\mathrm{WR} / \mathrm{O}$ mass ratios.

We then computed binary evolution models including the physics of rotation in both stellar components as well as the spin-up process of the mass gainer due to angular momentum accretion. In these models, the surface of the accreting star is continuously spun-up by accretion, while at the same time angular momentum is transported from the outer layers into the stellar interior by rotationally induced mixing processes.
By employing a simple model for the mass loss of rapidly rotating luminous stars - the so called $\Omega$-limit, which was actually worked out to describe the mass loss processes in Luminous Blue Variables (Langer 1997) - accretion is drastically reduced once the star reaches critical rotation at its surface. The mass accretion rate is then controlled by the time scale of internal angular momentum transport.

Some first such model for Case A and early Case B have been computed by Wellstein (Langer et al. 2003, 2004) for a primary mass of $15 M_{\odot}$ and a mass ratio close to one. The result was that rather high mass accretion efficiencies $(\beta \simeq 0.7)$ could be obtained for initial periods shorter than about 8 days. Here we find that, with the same physical assumptions although at higher system mass, the accretion efficiency drops to about $10 \%$ at an initial mass ratio of 1.7. As Wellstein (2001) computed one early Case A model for a $26 M_{\odot}+25 M_{\odot}$ system which gave $\beta=0.63$, it is like the high initial mass ratio in our models which is responsible for the low accretion efficiency: larger initial mass ratios lead to larger mass transfer rates and, as the time scale of internal angular momentum transport in the accreting star is rather unaffected, to smaller accretion efficiencies.

Our rotating models - in which the accretion efficiency is no free parameter any more but is computed selfconsistent and time-dependent - reproduce the observed $\mathrm{WR}+\mathrm{O}$ binaries quite well, i.e. as good as our models without rotation physics, where the accretion efficiency is a free parameter. Our simplified considerations in Sect. 3 have shown that this is unlikely attributable to the freedom in the choice of the initial parameter of the binary system, i.e. initial masses and period - at least under the assumption that contact was avoided. In case of contact, various new parameters enter the model, similar to the case of common envelope evolution. And indeed, also our rotating models can not reproduce HD 211853 very well, mostly because it currently has a too short orbital period, which was likely even significantly shorter at the beginning of its $\mathrm{WR}+\mathrm{O}$ stage. However, this of course only confirms the result of the simpler approaches that a contact-free approach does not work well for this system.

In summary we can say that the system mass and angular momentum loss model used here - which is the first detailed approach to tackle the long-standing angular momentum problem in mass transferring binaries - has passed the test of $\mathrm{WR}+\mathrm{O}$ binaries. However, it still needs to be explored over which part of the space spanned by the initial binary parameters this model works well, and to what extent its results are sensitive to future improvements in the stellar interior physics. The inclusion of magnetic fields generated by differential rotation (Spruit 2002) will be the next step in this direction (Petrovic et al. 2004).

\section{References}

Bonanos, A. Z., Stanek, K. Z., Udalski, A., et al. 2004, ApJ, 611, L33 Braun, A. 1998, Ph.D. Thesis

Braun, H., \& Langer, N. 1995, A\&A, 297, 483

Brookshaw, L., \& Tavani, M. 1993, ApJ, 410, 719

Chevalier, C., \& Ilovaisky, S. A. 1998, A\&A, 330, 201 
de Loore, C., \& de Greve, J. P. 1992, A\&AS, 94, 453

Demers, H., Moffat, A. F. J., Marchenko, S. V., Gayley, K. G., \& Morel, T. 2002, ApJ, 577, 409

Dessart, L., Langer, N., \& Petrovic, J. 2003, A\&A, 404, 991

Hamann, W.-R., \& Koesterke, L. 1998, A\&A, 335, 1003

Hamann, W.-R., Koesterke, L., \& Wessolowski, U. 1995, A\&A, 299, 151

Heger, A., \& Langer, N. 2000, ApJ, 544, 1016

Heger, A., Langer, N., \& Woosley, S. E. 2000, ApJ, 528, 368

Heger, A., Woosley, S. E., Langer, N., \& Spruit, H. C. 2004, in IAU Symp., 215, 591

Iglesias, C. A., \& Rogers, F. J. 1996, ApJ, 464, 943

King, A. R., Schenker, K., Kolb, U., \& Davies, M. B. 2001, MNRAS, 321,327

Kippenhahn, R., \& Thomas, H.-C. 1970, in Stellar Rotation, IAU Coll., 4, 20

Kippenhahn, R., Kohl, K., \& Weigert, A. 1967, Z. Astrophys., 66, 58

Kopal, Z., ed. 1978, Dynamics of Close Binary Systems

Kudritzki, R. P., Pauldrach, A., Puls, J., \& Abbott, D. C. 1989, A\&A, 219, 205

Lamontagne, R., Moffat, A. F. J., Drissen, L., Robert, C., \& Matthews, J. M. 1996, AJ, 112, 2227

Langer, N. 1991, A\&A, 252, 669

Langer, N. 1997, in Luminous Blue Variables: Massive Stars in Transition, ASP Conf. Ser., 120, 83

Langer, N. 1998, A\&A, 329, 551

Langer, N., Deutschmann, A., Wellstein, S., \& Höflich, P. 2000, A\&A, 362,1046

Langer, N., Wellstein, S., \& Petrovic, J. 2003, in IAU Symp., 212, 275

Langer, N., Yoon, S.-C., Petrovic, J., \& Heger, A. 2004, in IAU Symp., 215,535

Maeder, A., \& Meynet, G. 2003, A\&A, 411, 543
Massey, P. 1981, ApJ, 244, 157

Massey, P., \& Grove, K. 1989, ApJ, 344, 870

Meyer, F., \& Meyer-Hofmeister, E. 1983, A\&A, 121, 29

Meynet, G., \& Maeder, A. 2000, A\&A, 361, 101

Niemela, V. S., \& Moffat, A. F. J. 1982, ApJ, 259, 213

Packet, W. 1981, A\&A, 102, 17

Paczyński, B. 1967, Acta Astron., 17, 355

Paczynski, B. 1991, ApJ, 370, 597

Panov, K. P., \& Seggewiss, W. 1990, A\&A, 227, 117

Petrovic, J., \& Langer, N. 2004, in Rev. Mex. Astron. Astrofis. Conf. Ser., 231

Petrovic, J., Langer, N., Yoon, S.-C., \& Heger, A. 2004, A\&A, accepted

Podsiadlowski, P., Joss, P. C., \& Hsu, J. J. L. 1992, ApJ, 391, 246

Rauw, G., De Becker, M., Nazé, Y., et al. 2004, A\&A, 420, L9

Ritter, H. 1988, A\&A, 202, 93

Spruit, H. C. 2002, A\&A, 381, 923

Underhill, A. B., Yang, S., \& Hill, G. M. 1988, PASP, 100, 1256

van den Heuvel, E. P. J., \& Heise, J. 1972, Nature Phys. Sci., 239, 67

van der Hucht, K. A. 2001, New Astron. Rev., 45, 135

Vanbeveren, D. 1982, A\&A, 105, 260

Vanbeveren, D. 1991, A\&A, 252, 159

Vanbeveren, D., de Greve, J. P., de Loore, C., \& van Dessel, E. L. 1979, A\&A, 73, 19

Wellstein, S. 2001, Ph.D. Thesis

Wellstein, S., \& Langer, N. 1999, A\&A, 350, 148

Wellstein, S., Langer, N., \& Braun, H. 2001, A\&A, 369, 939

Woosley, S. E., Heger, A. 2004, in IAU Symp., 215, 601

Yoon, S.-C., \& Langer, N. 2004a, A\&A, 419, 645

Yoon, S.-C., \& Langer, N. 2004b, A\&A, 419, 623

Zahn, J.-P. 1977, A\&A, 57, 383 